Article

\title{
Non-Targeted Metabolomic Analysis of Methanolic Extracts of Wild-Simulated and Field-Grown American Ginseng
}

\author{
Hongqiang Lin, Hailin Zhu, Jing Tan, Han Wang, Qinghai Dong, Fulin Wu, Yunhe Liu, \\ Pingya Li ${ }^{*}$ and Jinping Liu *(D) \\ Research Center of Natural Drug, School of Pharmaceutical Sciences, Jilin University, Changchun 130021, China; \\ linhq17@mails.jlu.edu.cn (H.L.); 13578965875@163.com (H.Z.); tanjing17@mails.jlu.edu.cn (J.T.); \\ hanw17@mails.jlu.edu.cn (H.W.); dongqh17@mails.jlu.edu.cn (Q.D.); wufl17@mails.jlu.edu.cn (F.W.); \\ lyh133700@163.com (Y.L.) \\ * Correspondence: lipy@jlu.edu.cn (P.L.); liujp@jlu.edu.cn (J.L.); Tel.: +86-431-85619803 (P.L.)
}

Received: 10 February 2019; Accepted: 14 March 2019; Published: 18 March 2019

\begin{abstract}
Aiming at revealing the structural diversity of secondary metabolites and the different patterns in wild-simulated American ginseng (WsAG) and field-grown American ginseng (FgAG), a comprehensive and unique phytochemical profile study was carried out. In the screening analysis, a total of 121 shared compounds were characterized in FgAG and WsAG, respectively. The results showed that both of these two kinds of American ginseng were rich in natural components, and were similar in terms of the kinds of compound they contained. Furthermore, in non-targeted metabolomic analysis, when taking the contents of the constituents into account, it was found that there indeed existed quite a difference between FgAG and WsAG, and 22 robust known biomarkers enabling the differentiation were discovered. For WsAG, there were 12 potential biomarkers including two ocotillol-type saponins, two steroids, six damarane-type saponins, one oleanane-type saponins and one other compound. On the other hand, for FgAG, there were 10 potential biomarkers including two organic acids, six damarane-type saponins, one oleanane-type saponin, and one ursane. In a word, this study illustrated the similarities and differences between FgAG and WsAG, and provides a basis for explaining the effect of different growth environments on secondary metabolites.
\end{abstract}

Keywords: wild-simulated American ginseng; field-grown American ginseng; screening analysis; metabolomic analysis

\section{Introduction}

American ginseng (Panax quinquefolius L.) is grouped into four categories: wild, wild-simulated, woods-grown, and field-grown [1,2]. The herb growing in its native habitat is called wild American ginseng. Wild-simulated American ginseng (WsAG) refers to a method of growing ginseng in a hardwood forest environment under natural conditions without any other human intervention [2-4]. As such, WsAG roots are indeed indistinguishable from the wild roots due to the similar characteristics. When the seeds are planted in hardwood forests, and are grown in prepared rows or beds, or with removed ground vegetation or fertilizer and pesticides being available [5-7], it is called as wood-grown ginseng. This variety of American ginseng requires 6 to 9 years to mature before harvesting [8]. Different from wild-simulated category, the quality of wood-grown ginseng is between that of the wild and field-grown categories. That means, wood-grown American ginseng cannot be considered a substitute of the wild one. Field-grown American ginseng (FgAG), also called cultivated American ginseng, is intensely cultivated under artificial shade structures using fertilizers and pesticides $[4,9]$. 
Generally speaking, FgAG is harvested after 3-4 years, while WsAG is collected at least after 10-20 years or longer [10].

Modern pharmacological studies have shown that American ginseng has immunomodulatory [11], anti-tumor [12], anti-fatigue [13,14], anti-diabetic [15-17], anti-oxidant effects [18] and the functions of improving impaired memory and learning functions [14,19], etc. Furthermore, it is the traditional belief that roots from the wild are more medicinally efficacious, more potent and more valuable than those from cultivated sources, and wild roots thus command much higher prices on the Chinese medicine market [20-22]. But, since the late 18th century, natural wild American ginseng resources suffered a sharp decline due to predatory exploitation in North America under the influence of economic interests, and are nearing extinction now [23]. Meanwhile WsAG, with high quality and four to ten times the retail value of field-grown roots, is similar to the wild one [4]. Actually, planting wild-simulated ginseng is encouraged with the aim of reducing harvest pressure on wild populations [24]. Wild and wild-simulated roots could share the same export and trade regulations due to the similar morphology phenotype and market value $[8,25]$. Recently, because the so-called wild-simulated American ginseng could capitalize on the premium paid for wild-appearing roots, and the species appeared well suited to the practice of forest farming, American ginseng has been recommended as an agroforestry crop candidate [26]. With the continuous expansion of the folk and clinical applications of American ginseng, it is necessary to conduct an in-depth study on the chemical constituents of American ginseng aiming to clarify the material basis of efficacy. So far, there are a few comparative analysis on FgAG and wild American ginseng $[27,28]$. These results showed that ginsenosides are different between them [26], especially, the ratios of $\mathrm{Rg}_{1} / \mathrm{Rd}, \mathrm{Rg}_{1} / \mathrm{Rb}_{1}$ and $\left(\mathrm{Rg}_{1}+\mathrm{Re}\right) / \mathrm{Rd}$ are characteristic markers for differentiating these two groups [28]. However, a comparative study on the chemical composition between FgAG and WsAG does not exist.

Untargeted metabolomics, being able to profile diverse classes of metabolites, has been successfully applied to compare and identify the overall small-molecule components of different groups of samples [29]. Ultra-high performance liquid chromatography (UPLC) combined with quadrupole time-of-flight tandem mass spectrometry (QTOF-MS) and multivariate statistical analyses are often applied to profile the different groups. For multivariate statistical analyses, principal component analysis (PCA) and orthogonal partial least squares discriminant analysis (OPLS-DA) are the most common statistical methods. Meanwhile, UPLC-QTOF-MS combined with the automated data processing software UNIFI was often applied recently in the characterization of chemical components of herbal medicines [30-35]. When the coeluting constituents possessed different $m / z$ values, HR-MS can provide a specific and accurate mass. While, UNIFI, a highly comprehensive, high throughput, efficient and simple platform, offers a method for integrating data acquisition, data mining, library searching and reporting generation.

Aiming to find out the similarities and differences between FgAG and WsAG, and to provide a reference for quality control and material basis of efficacy, the screening and the comparative analysis of chemical constituents in FgAG and WsAG is conducted for the first time in this paper. The shared constituents would be evaluated with the UPLC-QTOF-MS method combined with UNIFI. The characteristic components were to be found using the untargeted metabolomics method. The results will also be helpful in explaining the different pharmacological activities and controlling the quality of FgAG and WsAG.

\section{Results}

\subsection{Identification of Components from FgAG and WsAG Based on the UNIFI Platform}

As a result of our screening analysis, a total of 121 compounds were identified or tentatively characterized in both positive and negative mode from FgAG and WsAG (Table 1), the base peak intensity (BPI) chromatograms are shown in Figure 1, and their chemical structures are shown in Figure 2. 
Table 1. Compounds identified from FgAG and WsAG by UPLC-QTOF-MS ${ }^{\mathrm{E}}$.

\begin{tabular}{|c|c|c|c|c|c|c|c|c|c|}
\hline No. & $t_{R}(\min )$ & Formula & $\begin{array}{l}\text { Calculated } \\
\text { Mass (Da) }\end{array}$ & $\begin{array}{l}\text { TheoreticalMass } \\
\text { (Da) }\end{array}$ & $\begin{array}{l}\text { Mass Error } \\
(\mathrm{ppm})\end{array}$ & $\mathrm{MS}^{\mathrm{E}}$ Fragmentation & Identification & Sources & Ref. \\
\hline 1 & 0.57 & $\mathrm{C}_{7} \mathrm{H}_{12} \mathrm{O}_{6}$ & 192.0636 & 192.0634 & 1.0 & $\begin{array}{l}191.0563[\mathrm{M}-\mathrm{H}]^{-}, 173.0454\left[\mathrm{M}-{\left.\mathrm{H}-\mathrm{H}_{2} \mathrm{O}\right]^{-},}_{127.0407[\mathrm{M}}\right. \\
\left.-\mathrm{H}-\mathrm{H}_{2} \mathrm{O}-\mathrm{HCOOH}\right]^{-}, 109.0452[\mathrm{M}- \\
\left.\mathrm{H}-2 \mathrm{H}_{2} \mathrm{O}-\mathrm{HCOOH}\right]^{-}, 91.0352\left[\mathrm{M}-\mathrm{H}-3 \mathrm{H}_{2} \mathrm{O}-\mathrm{HCOOH}\right]^{-}\end{array}$ & Quinic acid & WsAG, FgAG & $\mathrm{s}$ \\
\hline 2 & 0.64 & $\mathrm{C}_{12} \mathrm{H}_{22} \mathrm{O}_{11}$ & 342.1162 & 342.1165 & 0.8 & $341.1092[\mathrm{M}-\mathrm{H}]^{-}, 179.0562[\mathrm{M}-\mathrm{H}-\mathrm{Glu}]^{-}$ & $\alpha$-Maltose & WsAG, FgAG & $\mathrm{s}$ \\
\hline 3 & 0.77 & $\mathrm{C}_{10} \mathrm{H}_{13} \mathrm{~N}_{5} \mathrm{O}_{4}$ & 267.0959 & 267.0968 & -3.3 & $\begin{array}{l}268.1031[\mathrm{M}+\mathrm{H}]^{+}, 237.0874\left[\mathrm{M}+\mathrm{H}-\mathrm{CH}_{2} \mathrm{OH}\right]^{+}, 226.0898[\mathrm{M} \\
\left.+\mathrm{H}^{-} \mathrm{CN}_{2} \mathrm{H}_{2}\right]^{+}, 136.0612[\mathrm{M}+\mathrm{H}-\mathrm{Rib}]^{+}, 130.0495[\mathrm{M}+ \\
\left.\mathrm{H}-\mathrm{CH}_{2} \mathrm{OH}-\mathrm{C}_{4} \mathrm{~N}_{4} \mathrm{H}_{3}\right]^{+}\end{array}$ & Adenosine & WsAG, FgAG & $\mathrm{s}$ \\
\hline 4 & 0.93 & $\mathrm{C}_{12} \mathrm{H}_{22} \mathrm{O}_{11}$ & 342.1168 & 342.1162 & 1.7 & $\begin{array}{l}\text { 341.1095[M - H }]^{-}, 287.1097\left[\mathrm{M}-{\left.\mathrm{H}-3 \mathrm{H}_{2} \mathrm{O}\right]^{-}}\right. \\
179.0563\left[\mathrm{M}-\mathrm{H}^{-G l u}\right]^{-}\end{array}$ & Sucrose & WsAG, FgAG & $\mathrm{s}$ \\
\hline 5 & 0.95 & $\mathrm{C}_{9} \mathrm{H}_{11} \mathrm{NO}_{2}$ & 165.0777 & 165.0782 & -3.0 & $\begin{array}{l}166.0850[\mathrm{M}+\mathrm{H}]^{+}, 150.0589\left[\mathrm{M}+\mathrm{H}-\mathrm{NH}_{2}\right]^{+}, 132.0486[\mathrm{M}+ \\
\left.\mathrm{H}-\mathrm{H}_{2} \mathrm{O}-\mathrm{NH}_{2}\right]^{+}, 120.0807[\mathrm{M}+\mathrm{H}-\mathrm{HCOOH}]^{+}, 91.0559[\mathrm{M}+ \\
\text { H-CH-NH } \\
\text { H }-\mathrm{HCOOH}]^{+}\end{array}$ & L-Phenylalanine & WsAG, FgAG & $\mathrm{s}$ \\
\hline $6^{*}$ & 1.02 & $\mathrm{C}_{14} \mathrm{H}_{18} \mathrm{O}_{10}$ & 346.0903 & 346.0900 & 1.0 & $\begin{array}{l}345.0830[\mathrm{M}-\mathrm{H}]^{-}, 327.0598\left[\mathrm{M}-\mathrm{H}_{-} \mathrm{H}_{2} \mathrm{O}\right]^{-}, 309.0728[\mathrm{M} \\
-\mathrm{H}-2 \mathrm{H}_{2} \mathrm{O}^{-},, 165.0195[\mathrm{M}-\mathrm{H}-\mathrm{Glu}]^{-}, 150.0115[\mathrm{M}- \\
\left.\mathrm{H}-\mathrm{Glu}_{-} \mathrm{CH}_{3}\right]^{-}\end{array}$ & Methyl gallate 3-O- $\beta$-D-glucoside & $\begin{array}{l}\text { WsAG > FgAG } \\
\text { VIP: } 14.18 \\
p<0.001\end{array}$ & $\mathrm{~s}$ \\
\hline 7 & 1.51 & $\mathrm{C}_{11} \mathrm{H}_{12} \mathrm{~N}_{2} \mathrm{O}_{2}$ & 204.0899 & 204.0899 & -0.1 & $\begin{array}{l}203.0826[\mathrm{M}-\mathrm{H}]^{-}, 141.0660\left[\mathrm{M}-\mathrm{H}-\mathrm{HCOOH}-\mathrm{NH}_{2}\right]^{-} \\
129.0506\left[\mathrm{M}-\mathrm{H}-\mathrm{C}_{3} \mathrm{H}_{6} \mathrm{O}_{2}\right]^{-}\end{array}$ & L-Tryptophane & WsAG, FgAG & $\mathrm{s}$ \\
\hline 8 & 4.71 & $\mathrm{C}_{17} \mathrm{H}_{20} \mathrm{O}_{9}$ & 368.1106 & 368.1107 & -0.4 & 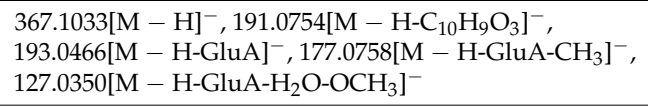 & 3-O-trans-Feruloylquinic Acid & WsAG, FgAG & [36] \\
\hline 9 & 4.92 & $\mathrm{C}_{48} \mathrm{H}_{82} \mathrm{O}_{19}$ & 962.5440 & 962.5450 & -1.0 & 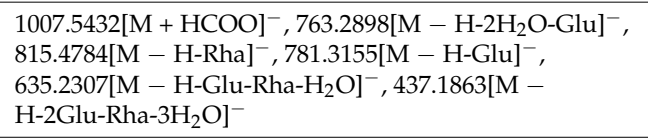 & Majoroside $\mathrm{F}_{6}$ & WsAG, FgAG & [37] \\
\hline 10 & 5.18 & $\mathrm{C}_{36} \mathrm{H}_{58} \mathrm{O}_{8}$ & 618.4130 & 618.4132 & -0.3 & $\begin{array}{l}\text { 619.4203[M + H }{ }^{+}, 439.3712[\mathrm{M}+\mathrm{H}-\mathrm{Glu}]^{+}, 422.3451[\mathrm{M}+ \\
\mathrm{H}-\mathrm{Glu}-\mathrm{OH}]^{+}, 383.2823\left[\mathrm{M}+\mathrm{H}-\mathrm{Glu}-\mathrm{C}_{4} \mathrm{H}_{8}\right]^{+}, 297.2336[\mathrm{M}+ \\
\left.\text { H-Glu- } \mathrm{C}_{9} \mathrm{H}_{16} \mathrm{O}\right]^{+}\end{array}$ & $\begin{array}{l}\text { Oleanolic acid } \\
-28-O-\beta \text {-D-glucopyranoside }\end{array}$ & WsAG, FgAG & $\mathrm{s}$ \\
\hline 11 & 5.32 & $\mathrm{C}_{48} \mathrm{H}_{82} \mathrm{O}_{20}$ & 978.5399 & 978.5397 & -0.3 & 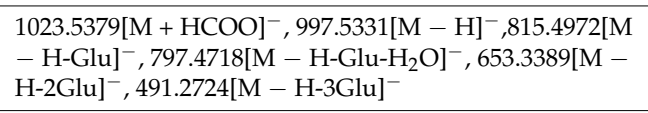 & Yesanchinoside B & WsAG, FgAG & [38] \\
\hline $12 *$ & 5.54 & $\mathrm{C}_{47} \mathrm{H}_{80} \mathrm{O}_{19}$ & 948.5305 & 948.5294 & 1.2 & 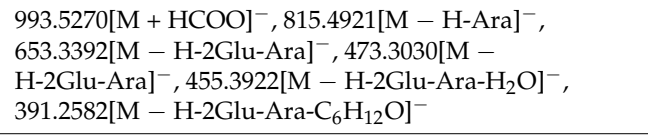 & Yesanchinoside C & $\begin{array}{l}\text { WsAG }>\text { FgAG } \\
\text { VIP: } 6.18 \\
p<0.001\end{array}$ & {$[38]$} \\
\hline $13^{\#}$ & 5.78 & $\mathrm{C}_{48} \mathrm{H}_{82} \mathrm{O}_{19}$ & 962.5443 & 962.5450 & -1.0 & 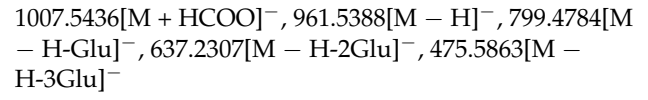 & Notoginsenoside N & $\begin{array}{l}\text { WsAG }<\text { FgAG } \\
\text { VIP: } 11.83 \\
p<0.001\end{array}$ & [39] \\
\hline
\end{tabular}


Table 1. Cont

\begin{tabular}{|c|c|c|c|c|c|c|c|c|c|}
\hline No. & $t_{R}(\min )$ & Formula & $\begin{array}{l}\text { Calculated } \\
\text { Mass (Da) }\end{array}$ & $\begin{array}{l}\text { TheoreticalMass } \\
\text { (Da) }\end{array}$ & $\begin{array}{l}\text { Mass Error } \\
(\mathrm{ppm})\end{array}$ & $\mathrm{MS}^{\mathrm{E}}$ Fragmentation & Identification & Sources & Ref. \\
\hline $14^{\#}$ & 5.94 & $\mathrm{C}_{42} \mathrm{H}_{74} \mathrm{O}_{15}$ & 818.5031 & 818.5028 & 0.4 & $\begin{array}{l}\text { 863.5006[M + HCOO }]^{-}, 667.4323[\mathrm{M}-\mathrm{H}-\mathrm{Rh}]^{-} \\
533.2329\left[\mathrm{M}-\mathrm{H}^{-} \mathrm{C}_{6} \mathrm{H}_{13} \mathrm{O}_{2}-\mathrm{C}_{11} \mathrm{H}_{19} \mathrm{O}\right]^{-}, 506.3845[\mathrm{M}- \\
\text { H-Glu-Rha] }]^{-}, 477.2169\left[\mathrm{M}-\mathrm{H}^{-} \mathrm{C}_{20} \mathrm{H}_{36} \mathrm{O}_{4}\right]^{-}\end{array}$ & Quinquenoside $\mathrm{L}_{9}$ & $\begin{array}{c}\text { WsAG }<\text { FgAG } \\
\text { VIP: } 7.20 \\
p=0.0002\end{array}$ & $\mathrm{~s}$ \\
\hline 15 & 5.94 & $\mathrm{C}_{42} \mathrm{H}_{72} \mathrm{O}_{14}$ & 800.4915 & 800.4922 & -0.9 & $\begin{array}{l}\text { 801.4988[M + H] }]^{+}, 621.4983\left[\mathrm{M}+\mathrm{H}-\mathrm{Glu}-\mathrm{H}_{2} \mathrm{O}\right]^{+} \\
459.3659[\mathrm{M}+\mathrm{H}-2 \mathrm{Glu}]^{+}, 423.3450\left[\mathrm{M}+\mathrm{H}-2 \mathrm{Glu}-3 \mathrm{H}_{2} \mathrm{O}\right]^{+}\end{array}$ & Majoroside $\mathrm{F}_{2}$ & WsAG, FgAG & [40] \\
\hline 16 & 6.25 & $\mathrm{C}_{26} \mathrm{H}_{34} \mathrm{O}_{11}$ & 522.2097 & 522.2101 & -0.7 & $\begin{array}{l}\text { 567.2079[M + HCOO }]^{-}, 521.2204[\mathrm{M}-\mathrm{H}]^{-}, 458.2935[\mathrm{M} \\
\left.-\mathrm{H}-\mathrm{H}_{2} \mathrm{O}-\mathrm{C}_{2} \mathrm{H}_{5} \mathrm{O}\right]^{-}, 341.1396\left[\mathrm{M}-\mathrm{H}-\mathrm{Glu}-\mathrm{H}_{2} \mathrm{O}\right]^{-} \\
178.0559\left[\mathrm{M}-\mathrm{H}_{20}\right.\end{array}$ & Urolignoside & WsAG, FgAG & [41] \\
\hline 17 & 6.48 & $\mathrm{C}_{54} \mathrm{H}_{92} \mathrm{O}_{23}$ & 1108.6034 & 1108.6029 & 0.4 & 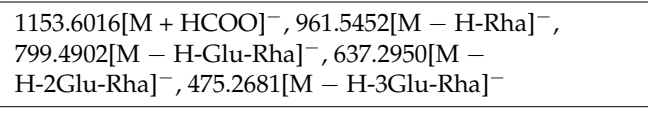 & Yesanchinoside E & WsAG, FgAG & [42] \\
\hline 18 & 6.77 & $\mathrm{C}_{47} \mathrm{H}_{80} \mathrm{O}_{18}$ & 932.5348 & 932.5345 & 0.3 & 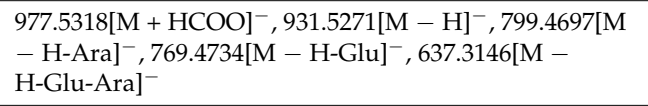 & Quinquenoside $\mathrm{F}_{6}$ & WsAG, FgAG & [37] \\
\hline 19 & 6.88 & $\mathrm{C}_{48} \mathrm{H}_{82} \mathrm{O}_{19}$ & 962.5450 & 962.5450 & -0.1 & 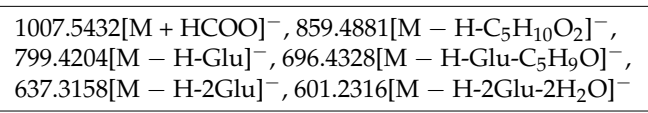 & Quinquenoside $\mathrm{L}_{2}$ & WsAG, FgAG & [43] \\
\hline 20 & 7.01 & $\mathrm{C}_{42} \mathrm{H}_{72} \mathrm{O}_{14}$ & 800.4914 & 800.4922 & -1.0 & 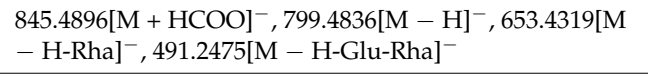 & (24S)-Pseudoginsenoside $F_{11}$ & WsAG, FgAG & s \\
\hline 21 * & 7.05 & $\mathrm{C}_{47} \mathrm{H}_{80} \mathrm{O}_{18}$ & 932.5349 & 932.5345 & 0.4 & $\begin{array}{l}\text { 977.5346[M + HCOO] }{ }^{-}, 840.4930\left[\mathrm{M}-\mathrm{H}-2 \mathrm{H}_{2} \mathrm{O}^{-} \mathrm{C}_{4} \mathrm{H}_{7}\right]^{-} \\
799.4859[\mathrm{M}-\mathrm{H}-\mathrm{Hyl}]^{-}, 769.4735[\mathrm{M}-\mathrm{H}-\mathrm{Glu}]^{-} \\
637.4321\left[\mathrm{M}-{\mathrm{H}-\mathrm{Glu}-\mathrm{Xyl}]^{-}}^{-}\right.\end{array}$ & Notoginsenoside $\mathrm{R}_{1}$ & $\begin{array}{l}\text { WsAG > FgAG } \\
\text { VIP: } 24.59 \\
p<0.001\end{array}$ & $\mathrm{~s}$ \\
\hline $22 *$ & 7.10 & $\mathrm{C}_{28} \mathrm{H}_{48} \mathrm{O}$ & 400.3723 & 400.3705 & 4.3 & $\begin{array}{l}423.3620[\mathrm{M}+\mathrm{Na}]^{+}, 382.2862\left[\mathrm{M}+\mathrm{H}-\mathrm{CH}_{3}\right]^{+}, 339.2934[\mathrm{M}+ \\
\left.\mathrm{H}-\mathrm{H}_{2} \mathrm{O}-\mathrm{C}_{3} \mathrm{H}_{7}\right]^{+}, 255.2948\left[\mathrm{M}+\mathrm{H}-\mathrm{H}_{2} \mathrm{O}-\mathrm{C}_{9} \mathrm{H}_{19}\right]^{+}\end{array}$ & Methylcholesta-7-en-3 $\beta$-ol & $\begin{array}{l}\text { WsAG }>\text { FgAG } \\
\text { VIP: } 4.69 \\
p<0.001\end{array}$ & [44] \\
\hline 23 & 7.12 & $\mathrm{C}_{48} \mathrm{H}_{82} \mathrm{O}_{19}$ & 962.5435 & 962.5450 & -1.5 & $\begin{array}{l}\text { 1007.54171[M + HCOO }]^{-}, 961.5329\left[\mathrm{M}-\mathrm{H}^{-}\right. \\
\text {799.3722[M - H-Glu] }{ }^{-}, 637.4321[\mathrm{M}-\mathrm{H}-2 \mathrm{Glu}]^{-} \\
475.3722[\mathrm{M}-\mathrm{H}-3 \mathrm{Glu}]^{-}, 391.4833\left[\mathrm{M}-\mathrm{H}-3 \mathrm{Glu}-\mathrm{C}_{6} \mathrm{H}_{12}\right]^{-}\end{array}$ & Notoginsenoside $\mathrm{R}_{6}$ & WsAG, FgAG & [42] \\
\hline 24 & 7.25 & $\mathrm{C}_{47} \mathrm{H}_{80} \mathrm{O}_{18}$ & 932.5335 & 932.5345 & -1.0 & 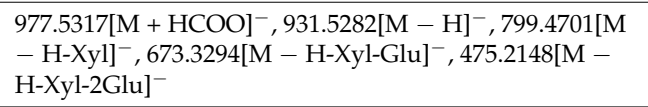 & Notoginsenoside ST5 & WsAG, FgAG & [45] \\
\hline 25 & 7.29 & $\mathrm{C}_{54} \mathrm{H}_{90} \mathrm{O}_{24}$ & 1122.5818 & 1122.5822 & 0.4 & $\begin{array}{l}\text { 1167.5812[M + HCOO }]^{-}, 1121.5747\left[\mathrm{M}-\mathrm{H}^{-}\right. \\
\text {959.5120[M - H-Glu }]^{-}, 797.4669[\mathrm{M}-\mathrm{H}-2 \mathrm{Glu}]^{-} \\
473.4334[\mathrm{M}-\mathrm{H}-4 \mathrm{Glu}]^{-}\end{array}$ & Quinquenoside IV & WsAG, FgAG & [42] \\
\hline 26 & 7.40 & $\mathrm{C}_{42} \mathrm{H}_{72} \mathrm{O}_{14}$ & 800.4918 & 800.4922 & -0.5 & $\begin{array}{l}\text { 845.4900[M + HCOO }]^{-}, 784.4683\left[\mathrm{M}-{\left.\mathrm{H}-\mathrm{CH}_{3}\right]^{-}},\right. \\
637.4340[\mathrm{M}-\mathrm{H}-\mathrm{Glu}]^{-}, 471.3787\left[\mathrm{M}-\mathrm{H}-2 \mathrm{Glu}^{-}\right.\end{array}$ & Ginsenoside $\mathrm{Rg}_{1}$ & WsAG, FgAG & $\mathrm{s}$ \\
\hline
\end{tabular}


Table 1. Cont.

\begin{tabular}{|c|c|c|c|c|c|c|c|c|c|}
\hline No. & $t_{R}(\min )$ & Formula & $\begin{array}{l}\text { Calculated } \\
\text { Mass (Da) }\end{array}$ & $\begin{array}{l}\text { TheoreticalMass } \\
\text { (Da) }\end{array}$ & $\begin{array}{l}\text { Mass Error } \\
\text { (ppm) }\end{array}$ & $\mathrm{MS}^{\mathrm{E}}$ Fragmentation & Identification & Sources & Ref. \\
\hline 27 & 7.47 & $\mathrm{C}_{48} \mathrm{H}_{82} \mathrm{O}_{18}$ & 946.5491 & 946.5501 & -1.0 & $\begin{array}{l}\text { 991.5473[M + HCOO }{ }^{-}, 945.5413\left[\mathrm{M}-\mathrm{H}^{-}, \text {, 783.5142[M }\right. \\
\text { - H-Glu] }{ }^{-}, 637.4125\left[\mathrm{M}-\mathrm{H}^{-G l u-R h a}\right]^{-}, 475.5147[\mathrm{M}- \\
\text { H-2Glu-Rha] }\end{array}$ & Ginsenoside Re & WsAG, FgAG & $\mathrm{s}$ \\
\hline 28 & 7.47 & $\mathrm{C}_{28} \mathrm{H}_{48} \mathrm{O}$ & 400.3715 & 400.3705 & 2.4 & $\begin{array}{l}423.3617[\mathrm{M}+\mathrm{Na}]^{+}, 401.3540\left[\mathrm{M}+\mathrm{H}^{+}, 383.2861[\mathrm{M}+\right. \\
\left.\mathrm{H}-\mathrm{H}_{2} \mathrm{O}\right]^{+}, 325.2982\left[\mathrm{M}+\mathrm{H}-\mathrm{H}_{2} \mathrm{O}-\mathrm{CH}_{3}-\mathrm{C}_{3} \mathrm{H}_{7}\right]^{+} \\
284.1420\left[\mathrm{M}+\mathrm{H}-\mathrm{H}_{2} \mathrm{O}-\mathrm{C}_{7} \mathrm{H}_{15}\right]^{+}, 175.1221[\mathrm{M}+ \\
\left.\mathrm{H}-\mathrm{H}_{2} \mathrm{O}-\mathrm{C}_{15} \mathrm{H}_{28}\right]^{+}\end{array}$ & Campesterol & WsAG, FgAG & $\mathrm{a}$ \\
\hline 29 & 7.69 & $\mathrm{C}_{45} \mathrm{H}_{74} \mathrm{O}_{17}$ & 886.4926 & 886.4925 & -0.1 & $\begin{array}{l}\text { 885.4853[M - H }]^{-}, 799.4748\left[\mathrm{M}-\mathrm{H}_{-M a l}{ }^{-}, 637.4303[\mathrm{M}\right. \\
\left.-\mathrm{H}^{-M a l-G l u}\right]^{-}, 475.3751\left[\mathrm{M}-\mathrm{H}_{-} \text {Mal-2Glu] }\right.\end{array}$ & Malonyl-ginsenoside $\mathrm{Rg}_{1}$ & WsAG, FgAG & [46] \\
\hline 30 & 7.85 & $\mathrm{C}_{42} \mathrm{H}_{72} \mathrm{O}_{14}$ & 800.4929 & 800.4922 & 0.8 & 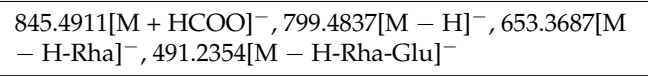 & Quinquenoside $\mathrm{L}_{11}$ & WsAG, FgAG & $\mathrm{s}$ \\
\hline $31^{\#}$ & 7.84 & $\mathrm{C}_{51} \mathrm{H}_{84} \mathrm{O}_{21}$ & 1032.5505 & 1032.5532 & 2.6 & 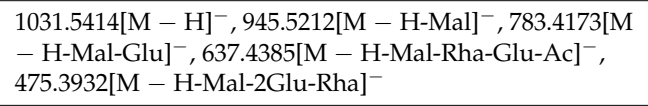 & Malonyl-ginsenoside Re & $\begin{array}{l}\text { WsAG }<\text { FgAG } \\
\text { VIP: } 5.65 \\
p=0.0060\end{array}$ & [39] \\
\hline 32 & 7.94 & $\mathrm{C}_{47} \mathrm{H}_{80} \mathrm{O}_{19}$ & 948.5282 & 948.5294 & -1.2 & $\begin{array}{l}\text { 947.5209[M - H] }{ }^{-}, 815.4786[\mathrm{M}-\mathrm{H}-\mathrm{Xyl}]^{-}, 653.2758[\mathrm{M} \\
\left.-\mathrm{H}_{-} \mathrm{Glu}-\mathrm{Xyl}\right]^{-}, 491.1787[\mathrm{M}-\mathrm{H}-2 \mathrm{Glu}-\mathrm{Xyl}]^{-}\end{array}$ & Vinaginsenoside $\mathrm{R}_{6}$ & WsAG, FgAG & [39] \\
\hline 33 & 8.15 & $\mathrm{C}_{47} \mathrm{H}_{80} \mathrm{O}_{17}$ & 916.5409 & 916.5396 & 1.4 & 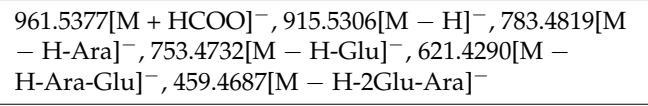 & Quinquenoside $\mathrm{L}_{14}$ & WsAG, FgAG & {$[47]$} \\
\hline 34 & 8.23 & $\mathrm{C}_{44} \mathrm{H}_{74} \mathrm{O}_{15}$ & 842.4996 & 842.5028 & -3.6 & 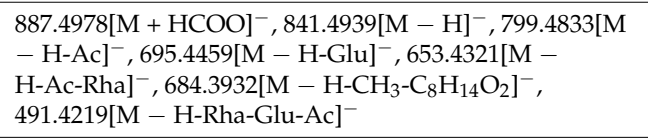 & Vinaginsenoside $R_{1}$ & WsAG, FgAG & [48] \\
\hline $35^{*}$ & 8.26 & $\mathrm{C}_{54} \mathrm{H}_{94} \mathrm{O}_{24}$ & 1126.6162 & 1126.6135 & 2.4 & $\begin{array}{l}\text { 1171.6110[M + } \mathrm{HCOO}^{-}, 1125.6094\left[\mathrm{M}-\mathrm{H}^{-}, 975.5349\right. \\
{\left[\mathrm{M}-\mathrm{H}^{-} \mathrm{Xyl}-\mathrm{H}_{2} \mathrm{O}\right]^{-}, 963.5502[\mathrm{M}-\mathrm{H}-\mathrm{Glu}]^{-}, 801.3547[\mathrm{M}} \\
-\mathrm{H}-2 \mathrm{Glu}]^{-}, 831.3214[\mathrm{M}-\mathrm{H}-\mathrm{Glu}-\mathrm{Xyl}]^{-}, 507.3214[\mathrm{M}- \\
\left.\mathrm{H}^{-3 G l u-X y l}\right]^{-}\end{array}$ & Quinquenoside $\mathrm{F}_{3}$ & $\begin{array}{l}\text { WsAG }>\text { FgAG } \\
\text { VIP: } 3.35 \\
p<0.001\end{array}$ & $\mathrm{~s}$ \\
\hline 36 & 8.57 & $\mathrm{C}_{44} \mathrm{H}_{74} \mathrm{O}_{15}$ & 842.5028 & 842.5012 & -1.7 & $\begin{array}{l}\text { 887.4995[M + HCOO] }{ }^{-}, 841.4939[\mathrm{M}-\mathrm{H}]^{-}, 637.4321[\mathrm{M} \\
\left.-\mathrm{H}^{-G l u-A c}\right]^{-}, 475.3030[\mathrm{M}-\mathrm{H}-2 \mathrm{Glu}-\mathrm{Ac}]^{-}, 391.4158[\mathrm{M} \\
\left.-\mathrm{H}^{-} 2 \mathrm{Glu}-\mathrm{Ac}-\mathrm{C}_{6} \mathrm{H}_{11}\right]^{-}\end{array}$ & Acetyl-Ginsenoside $\operatorname{Rg}_{1}$ & WsAG, FgAG & [39] \\
\hline $37^{*}$ & 8.59 & $\mathrm{C}_{41} \mathrm{H}_{70} \mathrm{O}_{13}$ & 770.4808 & 770.4816 & -1.0 & $\begin{array}{l}\text { 815.4798[M + } \mathrm{HCOO}^{-}, 769.4722\left[\mathrm{M}-\mathrm{H}^{-}\right]^{-}, 637.4321[\mathrm{M} \\
\left.-\mathrm{H}^{-A r a}\right]^{-}, 475.2678[\mathrm{M}-\mathrm{H}-\mathrm{Ara}-\mathrm{Glu}]^{-}, 391.1748[\mathrm{M}- \\
\left.\text { H-Ara-Glu- }_{6} \mathrm{H}_{11}\right]^{-}\end{array}$ & Notoginsenoside $\mathrm{R}_{2}$ & $\begin{array}{l}\text { WsAG }>\text { FgAG } \\
\text { VIP: } 4.83 \\
p<0.001\end{array}$ & [42] \\
\hline 38 & 8.63 & $\mathrm{C}_{48} \mathrm{H}_{82} \mathrm{O}_{19}$ & 962.5423 & 962.5450 & -2.7 & $\begin{array}{l}\text { 1007.5405[M + HCOO }]^{-}, 961.5371\left[\mathrm{M}-\mathrm{H}^{-}, 815.4317[\mathrm{M}\right. \\
\left.-\mathrm{H}^{-R h a}\right]^{-}, 799.4622[\mathrm{M}-\mathrm{H}-\mathrm{Glu}]^{-}, 653.4385[\mathrm{M}- \\
\left.\mathrm{H}^{-G l u-R h a}\right]^{-}, 617.4316\left[\mathrm{M}-\mathrm{H}-\mathrm{Hlu}-\mathrm{Rha}-\mathrm{H}_{2} \mathrm{O}\right]^{-} \\
\text {491.2912[M - H-2Glu-Rha] }]^{-}\end{array}$ & Majoroside $\mathrm{F}_{5}$ & WsAG, FgAG & {$[37]$} \\
\hline
\end{tabular}


Table 1. Cont

\begin{tabular}{|c|c|c|c|c|c|c|c|c|c|}
\hline No. & $t_{R}(\min )$ & Formula & $\begin{array}{l}\text { Calculated } \\
\text { Mass (Da) }\end{array}$ & $\begin{array}{l}\text { TheoreticalMass } \\
\text { (Da) }\end{array}$ & $\begin{array}{l}\text { Mass Error } \\
(\mathrm{ppm})\end{array}$ & $\mathrm{MS}^{\mathrm{E}}$ Fragmentation & Identification & Sources & Ref. \\
\hline 39 & 8.64 & $\mathrm{C}_{30} \mathrm{H}_{48} \mathrm{O}_{2}$ & 440.3646 & 440.3654 & -1.9 & $\begin{array}{l}441.3719[\mathrm{M}+\mathrm{H}]^{+}, 423.3606\left[\mathrm{M}+\mathrm{H}-\mathrm{H}_{2} \mathrm{O}\right]^{+}, 339.2908[\mathrm{M}+ \\
\left.\mathrm{H}-\mathrm{HCOOH}-\mathrm{C}_{4} \mathrm{H}_{8}\right]^{+}, 248.2948\left[\mathrm{M}+\mathrm{H}-\mathrm{C}_{14} \mathrm{H}_{24}\right]^{+} \\
203.1849\left[\mathrm{M}+\mathrm{H}-\mathrm{HCOOH}-\mathrm{C}_{14} \mathrm{H}_{24}\right]^{+}\end{array}$ & Deoxyoleanolic acid & WsAG, FgAG & [46] \\
\hline 40 & 8.78 & $\mathrm{C}_{48} \mathrm{H}_{80} \mathrm{O}_{18}$ & 944.5338 & 944.5345 & -0.6 & $\begin{array}{l}\text { 989.5320[M + HCOO }]^{-}, 943.5250\left[\mathrm{M}-\mathrm{H}^{-}, 781.4541[\mathrm{M}\right. \\
\left.-\mathrm{H}_{-} \mathrm{Glu}\right]^{-}, 619.4143[\mathrm{M}-\mathrm{H}-2 \mathrm{Glu}]^{-}, 457.5876[\mathrm{M}- \\
{\mathrm{H}-3 \mathrm{Glu}]^{-}}^{-}\end{array}$ & Quinquenoside $\mathrm{L}_{1}$ & WsAG, FgAG & [49] \\
\hline 41 & 8.83 & $\mathrm{C}_{53} \mathrm{H}_{88} \mathrm{O}_{23}$ & 1092.5718 & 1092.5716 & 0.2 & $\begin{array}{l}\text { 1137.5696[M + HCOO }]^{-}, 1091.5641\left[\mathrm{M}-\mathrm{H}^{-}\right. \\
\text {959.5571[M - H-Xyl] }{ }^{-}, 929.4601[\mathrm{M}-\mathrm{H}-\mathrm{Glu}]^{-} \\
\text {797.4852[M - H-Glu-Xyl] }\end{array}$ & Yesanchinoside G & WsAG, FgAG & [50] \\
\hline $42 *$ & 8.89 & $\mathrm{C}_{47} \mathrm{H}_{78} \mathrm{O}_{17}$ & 914.5225 & 914.5239 & -1.5 & 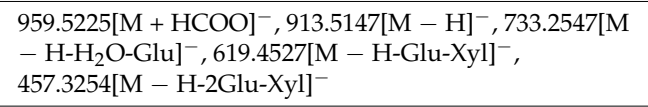 & Quinquenoside $\mathrm{L}_{8}$ & $\begin{array}{c}\text { WsAG > FgAG } \\
\text { VIP: } 3.97 \\
p=0.0004\end{array}$ & $\mathrm{~s}$ \\
\hline 43 & 8.97 & $\mathrm{C}_{48} \mathrm{H}_{82} \mathrm{O}_{19}$ & 962.5438 & 962.5450 & -1.2 & $\begin{array}{l}\text { 1007.5420[M + } \mathrm{HCOO}^{-}, 946.5212\left[\mathrm{M}-\mathrm{H}-\mathrm{CH}_{3}\right]^{-} \\
\left.\text {781.4533[M }-\mathrm{H}-\mathrm{Glu}-\mathrm{H}_{2} \mathrm{O}\right]^{-}, 637.4321[\mathrm{M}-\mathrm{H}-2 \mathrm{Glu}]^{-} \\
475.3932[\mathrm{M}-\mathrm{H}-3 \mathrm{Glu}]^{-}\end{array}$ & Majoroside $F_{1}$ & WsAG, FgAG & [40] \\
\hline $44^{*}$ & 9.14 & $\mathrm{C}_{42} \mathrm{H}_{70} \mathrm{O}_{13}$ & 782.4325 & 782.4816 & 1.2 & $\begin{array}{l}\text { 781.4747[M - H] }]^{-}, 619.4181\left[\mathrm{M}-\mathrm{H}_{-}-\mathrm{Glu}\right]^{-}, 457.4798[\mathrm{M} \\
-\mathrm{H}-2 \mathrm{Glu}]^{-}, 376.4797\left[\mathrm{M}-\mathrm{H}-2 \mathrm{Glu}^{-} \mathrm{C}_{6} \mathrm{H}_{9}\right]^{-}\end{array}$ & Quinquenoside $\mathrm{F}_{1}$ & $\begin{array}{l}\text { WsAG > FgAG } \\
\text { VIP: } 4.10 \\
p=0.0004\end{array}$ & [51] \\
\hline 45 & 9.27 & $\mathrm{C}_{15} \mathrm{H}_{10} \mathrm{O}_{6}$ & 286.0480 & 286.0477 & 0.8 & $\begin{array}{l}\text { 285.0407[M - H }]^{-}, 227.0521\left[\mathrm{M}-\mathrm{H}-\mathrm{C}_{2} \mathrm{H}_{2} \mathrm{O}_{2}\right]^{-} \\
\left.\text {151.0037[M }-\mathrm{H}-\mathrm{C}_{8} \mathrm{H}_{6} \mathrm{O}_{2}\right]^{-}, 106.0148\left[\mathrm{M}-\mathrm{H}-\mathrm{C}_{9} \mathrm{H}_{7} \mathrm{O}_{4}\right]^{-} \\
\left.\text {112.0351[M }-\mathrm{H}-\mathrm{C}_{9} \mathrm{H}_{5} \mathrm{O}_{5}\right]^{-}\end{array}$ & Kaempferol & WsAG, FgAG & $\mathrm{s}$ \\
\hline $46^{\#}$ & 9.32 & $\mathrm{C}_{53} \mathrm{H}_{86} \mathrm{O}_{24}$ & 1118.5514 & 1118.5509 & 0.5 & 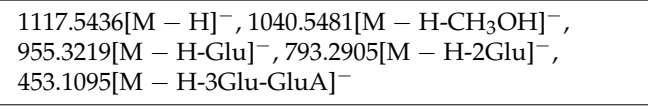 & Ginsenoside $\mathrm{R}_{\mathrm{OA}}$ & $\begin{array}{l}\text { WsAG }<\text { FgAG } \\
\text { VIP: } 12.60 \\
p<0.001\end{array}$ & [52] \\
\hline $47^{*}$ & 9.54 & $\mathrm{C}_{41} \mathrm{H}_{70} \mathrm{O}_{14}$ & 786.4775 & 786.4766 & 1.1 & $\begin{array}{l}\text { 831.4775[M + HCOO }]^{-}, 767.4297\left[\mathrm{M}-{\left.\mathrm{H}-\mathrm{H}_{2} \mathrm{O}\right]^{-}}\right. \\
\text {653.4318[M - H-Xyl }]^{-}, 491.2015\left[\mathrm{M}-\mathrm{H}^{-G l u-X y l}\right]^{-}\end{array}$ & Majonoside $\mathrm{R}_{2}$ & $\begin{array}{l}\text { WsAG > FgAG } \\
\text { VIP: } 25.80 \\
p<0.001\end{array}$ & [39] \\
\hline 48 & 9.61 & $\mathrm{C}_{48} \mathrm{H}_{80} \mathrm{O}_{19}$ & 960.5285 & 960.5294 & -0.9 & $\begin{array}{l}\text { 1005.5267[M + HCOO }]^{-}, 941.5316\left[\mathrm{M}-\mathrm{H}_{-} \mathrm{H}_{2} \mathrm{O}\right]^{-} \\
\text {797.4287[M - H-Glu] }]^{-}, 635.3221[\mathrm{M}-\mathrm{H}-2 \mathrm{Glu}]^{-} \\
473.2684[\mathrm{M}-\mathrm{H}-3 \mathrm{Glu}]^{-}\end{array}$ & Notoginsenoside G & WsAG, FgAG & [42] \\
\hline 49 & 9.68 & $\mathrm{C}_{42} \mathrm{H}_{72} \mathrm{O}_{14}$ & 800.4922 & 800.4922 & 0.0 & 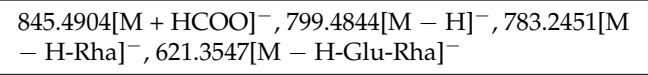 & Pseudo-ginsenoside $\mathrm{F}_{11}$ & WsAG, FgAG & $\mathrm{s}$ \\
\hline 50 & 9.73 & $\mathrm{C}_{36} \mathrm{H}_{62} \mathrm{O}_{10}$ & 654.4340 & 654.4343 & -0.4 & 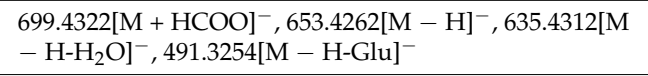 & Pseudo-ginsenoside $\mathrm{RT}_{5}$ & WsAG, FgAG & $\mathrm{s}$ \\
\hline 51 & 9.78 & $\mathrm{C}_{36} \mathrm{H}_{62} \mathrm{O}_{10}$ & 654.4337 & 654.4343 & -0.9 & $\begin{array}{l}\text { 655.4410[M + H }]^{+}, 599.4418\left[\mathrm{M}-\mathrm{H}-3 \mathrm{H}_{2} \mathrm{O}^{+}, 493.3437[\mathrm{M}\right. \\
\left.-\mathrm{H}^{+G l u}\right]^{+}, 457.2651\left[\mathrm{M}-\mathrm{H}-\mathrm{Glu}-2 \mathrm{H}_{2} \mathrm{O}\right]^{+}\end{array}$ & Pseudo-ginsenoside $\mathrm{RT}_{4}$ & WsAG, FgAG & [39] \\
\hline
\end{tabular}


Table 1. Cont

\begin{tabular}{|c|c|c|c|c|c|c|c|c|c|}
\hline No. & $t_{R}(\min )$ & Formula & $\begin{array}{l}\text { Calculated } \\
\text { Mass (Da) }\end{array}$ & $\begin{array}{l}\text { TheoreticalMass } \\
\text { (Da) }\end{array}$ & $\begin{array}{l}\text { Mass Error } \\
(\mathrm{ppm})\end{array}$ & $\mathrm{MS}^{\mathrm{E}}$ Fragmentation & Identification & Sources & Ref. \\
\hline 52 & 9.82 & $\mathrm{C}_{59} \mathrm{H}_{100} \mathrm{O}_{27}$ & 1240.6458 & 1240.6452 & 0.5 & $\begin{array}{l}\text { 1239.6380[M - H }]^{-}, 1107.6376\left[\mathrm{M}-\mathrm{H}-X y l^{-}\right. \\
\text {954.6930[M - H-Xyl-Glu }]^{-}, 783.4833\left[\mathrm{M}-\mathrm{H}^{-X y l-2 G l u}\right]^{-}, \\
621.4431[\mathrm{M}-\mathrm{H}-\mathrm{Xyl}-3 \mathrm{Glu}]^{-}, 459.4943[\mathrm{M}- \\
\mathrm{H}-X y l-4 G l u^{-}\end{array}$ & Ginsenoside $\mathrm{Ra}_{3}$ & WsAG, FgAG & [53] \\
\hline 53 & 9.96 & $\mathrm{C}_{41} \mathrm{H}_{70} \mathrm{O}_{13}$ & 770.4810 & 770.4816 & -0.8 & $\begin{array}{l}\text { 815.4804[M + HCOO }]^{-}, 751.4804\left[\mathrm{M}-{\left.\mathrm{H}-\mathrm{H}_{2} \mathrm{O}\right]^{-}}\right. \\
637.4904[\mathrm{M}-\mathrm{H}-\mathrm{Hra}]^{-}, 475.3804\left[\mathrm{M}-\mathrm{H}_{-}-\mathrm{Glu}_{-} \text {-Ara }\right]^{-}\end{array}$ & Ginsenoside $\mathrm{F}_{5}$ & WsAG, FgAG & [39] \\
\hline 54 & 9.98 & $\mathrm{C}_{58} \mathrm{H}_{98} \mathrm{O}_{26}$ & 1210.6350 & 1210.6346 & 0.3 & 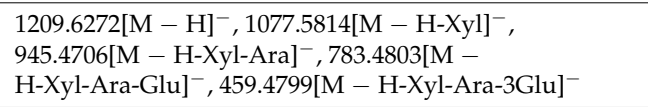 & Ginsenoside $\mathrm{Ra}_{2}$ & WsAG, FgAG & [53] \\
\hline 55 & 10.02 & $\mathrm{C}_{41} \mathrm{H}_{66} \mathrm{O}_{11}$ & 734.4590 & 734.4605 & -2.1 & 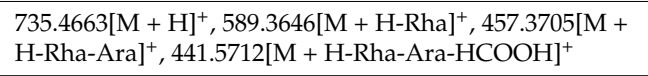 & Eleutheroside K & WsAG, FgAG & [54] \\
\hline 56 & 10.04 & $\mathrm{C}_{48} \mathrm{H}_{80} \mathrm{O}_{18}$ & 944.5320 & 944.5345 & -2.6 & 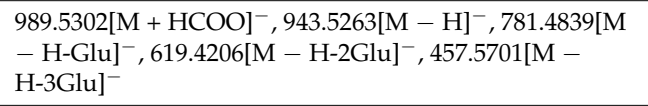 & Quinquenoside $\mathrm{L}_{6}$ & WsAG, FgAG & - \\
\hline 57 & 10.07 & $\mathrm{C}_{30} \mathrm{H}_{48} \mathrm{O}_{2}$ & 440.3638 & 440.3654 & -3.6 & $\begin{array}{l}441.3717[\mathrm{M}+\mathrm{H}]^{+}, 394.3508\left[\mathrm{M}+\mathrm{H}-\mathrm{H}_{2} \mathrm{O}-\mathrm{CHO}^{+}\right. \\
328.3504\left[\mathrm{M}+\mathrm{H}-\mathrm{CHO}-\mathrm{C}_{6} \mathrm{H}_{12}\right]^{+}, 219.1792[\mathrm{M}+ \\
\left.\mathrm{H}-\mathrm{C}_{15} \mathrm{H}_{26} \mathrm{O}\right]^{+}, 205.1619\left[\mathrm{M}+\mathrm{H}-\mathrm{H}_{2} \mathrm{O}-\mathrm{C}_{15} \mathrm{H}_{22} \mathrm{O}\right]^{+}\end{array}$ & $3 \beta$-Hydroxyolean-12-en-28-al & WsAG, FgAG & $\mathrm{a}$ \\
\hline 58 & 10.14 & $\mathrm{C}_{59} \mathrm{H}_{100} \mathrm{O}_{27}$ & 1240.6452 & 1240.6462 & 0.8 & $\begin{array}{l}\text { 1285.6444[M + HCOO }]^{-}, 1107.5976\left[\mathrm{M}-\mathrm{H}-\mathrm{Xyl}^{-}\right]^{-} \\
\text {945.4900[M - H-Xyl-Glu }]^{-}, 783.4835\left[\mathrm{M}-\mathrm{H}-\mathrm{Xyl}^{-} \mathrm{Glu}\right]^{-}, \\
459.4929[\mathrm{M}-\mathrm{H}-\mathrm{Hyl}-4 \mathrm{Glu}]^{-}\end{array}$ & Notoginsenoside Fa & WsAG, FgAG & [53] \\
\hline 59 & 10.24 & $\mathrm{C}_{54} \mathrm{H}_{92} \mathrm{O}_{23}$ & 1108.6039 & 1108.6029 & 0.8 & 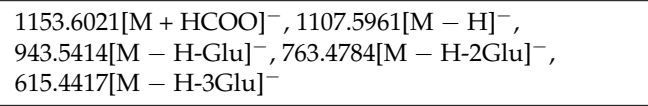 & Ginsenoside $\mathrm{Rb}_{1}$ & WsAG, FgAG & s \\
\hline 60 & 10.24 & $\mathrm{C}_{30} \mathrm{H}_{48} \mathrm{O}$ & 424.3692 & 424.3705 & -3.1 & $\begin{array}{l}425.3765[\mathrm{M}+\mathrm{H}]^{+}, 409.3102\left[\mathrm{M}+\mathrm{H}-\mathrm{H}_{2} \mathrm{O}\right]^{+}, 371.3759[\mathrm{M}+ \\
\left.\mathrm{H}-\mathrm{CH}_{3}-\mathrm{C}_{3} \mathrm{H}_{5}\right]^{+}, 189.1614\left[\mathrm{M}+\mathrm{H}-\mathrm{C}_{16} \mathrm{H}_{26} \mathrm{O}\right]^{+}, 205.1775[\mathrm{M} \\
\left.+\mathrm{H}_{-} \mathrm{C}_{15} \mathrm{H}_{26} \mathrm{O}\right]^{+}\end{array}$ & Olean-18-en-3-one & WsAG, FgAG & [55] \\
\hline 61 & 10.31 & $\mathrm{C}_{57} \mathrm{H}_{94} \mathrm{O}_{26}$ & 1194.6054 & 1194.6033 & 1.7 & 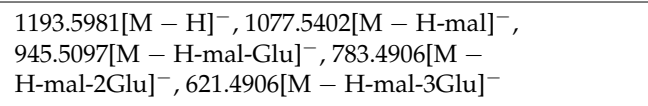 & Malonyl-ginsenoside $\mathrm{Rb}_{1}$ & WsAG, FgAG & [53] \\
\hline 62 & 10.33 & $\mathrm{C}_{42} \mathrm{H}_{72} \mathrm{O}_{13}$ & 784.4975 & 784.4973 & 0.3 & $\begin{array}{l}\text { 829.4957[M + HCOO }]^{-}, 768.4744\left[\mathrm{M}-{\left.\mathrm{H}-\mathrm{CH}_{3}\right]^{-}}\right. \\
635.4330[\mathrm{M}-\mathrm{H}-\mathrm{Rha}]^{-}, 471.3782\left[\mathrm{M}-\mathrm{H}^{-G l u-R h a}\right]^{-}\end{array}$ & 20(R)-Ginsenoside $\operatorname{Rg}_{2}$ & WsAG, FgAG & s \\
\hline 63 & 10.35 & $\mathrm{C}_{36} \mathrm{H}_{62} \mathrm{O}_{9}$ & 638.4391 & 638.4394 & -0.4 & 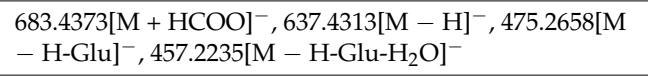 & 20(S)-Ginsenoside $\mathrm{Rh}_{1}$ & WsAG, FgAG & $\mathrm{s}$ \\
\hline 64 & 10.36 & $\mathrm{C}_{41} \mathrm{H}_{70} \mathrm{O}_{13}$ & 770.4817 & 770.4816 & 0.0 & $\begin{array}{l}\text { 815.4799[M + HCOO }]^{-}, 678.4450\left[\mathrm{M}-\mathrm{H}-2 \mathrm{H}_{2} \mathrm{O}-\mathrm{C}_{4} \mathrm{H}_{7}\right]^{-} \\
\text {637.4321[M - H-Ara }]^{-}, 590.2706\left[\mathrm{M}-\mathrm{H}-\mathrm{C}_{4} \mathrm{H}_{7}-\mathrm{C}_{9} \mathrm{H}_{16}\right]^{-} \\
475.2622[\mathrm{M}-\mathrm{H}-\mathrm{H} \text {-Glu-Ara }]^{-}\end{array}$ & Ginsenoside $\mathrm{F}_{3}$ & WsAG, FgAG & [39] \\
\hline
\end{tabular}


Table 1. Cont.

\begin{tabular}{|c|c|c|c|c|c|c|c|c|c|}
\hline No. & $t_{R}(\min )$ & Formula & $\begin{array}{l}\text { Calculated } \\
\text { Mass (Da) }\end{array}$ & $\begin{array}{l}\text { TheoreticalMass } \\
\text { (Da) }\end{array}$ & $\begin{array}{l}\text { Mass Error } \\
\text { (ppm) }\end{array}$ & $\mathrm{MS}^{\mathrm{E}}$ Fragmentation & Identification & Sources & Ref. \\
\hline 65 & 10.39 & $\mathrm{C}_{53} \mathrm{H}_{90} \mathrm{O}_{22}$ & 1078.5931 & 1078.5924 & 0.6 & $\begin{array}{l}\text { 1123.5913[M + } \mathrm{HCOO}^{-}, 943.5423\left[\mathrm{M}-\mathrm{H}_{-}-\mathrm{Araf}^{-}\right. \\
\left.\text {854.4890[M - } \mathrm{H}-\mathrm{H}_{2} \mathrm{O}-\mathrm{Araf}-\mathrm{C}_{4} \mathrm{H}_{7}\right]^{-}, 763.4850[\mathrm{M}- \\
\text { H-Glu-Araf }]^{-}\end{array}$ & Ginsenoside Rc & WsAG, FgAG & $\mathrm{s}$ \\
\hline 66 & 10.42 & $\mathrm{C}_{36} \mathrm{H}_{60} \mathrm{O}_{8}$ & 620.4276 & 620.4288 & -1.9 & $\begin{array}{l}621.4349[\mathrm{M}+\mathrm{H}]^{+}, 603.4238\left[\mathrm{M}+\mathrm{H}-\mathrm{H}_{2} \mathrm{O}\right]^{+}, 441.3714[\mathrm{M}+ \\
\mathrm{H}-\mathrm{Glu}]^{+}, 423.3612\left[\mathrm{M}+\mathrm{H}-\mathrm{Glu}-\mathrm{H}_{2} \mathrm{O}\right]^{+}, 350.2971[\mathrm{M}+ \\
\left.\text { H-Glu-2 } \mathrm{H}_{2} \mathrm{O}-\mathrm{C}_{4} \mathrm{H}_{7}\right]^{+}, 341.1160\left[\mathrm{M}+\mathrm{H}-\mathrm{Glu}^{+} \mathrm{C}_{6} \mathrm{H}_{12} \mathrm{O}\right]^{+}\end{array}$ & Ginsenoside $\mathrm{Rh}_{4}$ & WsAG, FgAG & [56] \\
\hline 67 & 10.46 & $\mathrm{C}_{58} \mathrm{H}_{98} \mathrm{O}_{26}$ & 1210.6353 & 1210.6346 & 0.6 & 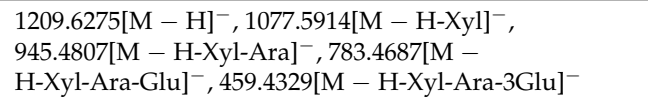 & Ginsenoside $\mathrm{Ra}_{1}$ & WsAG, FgAG & [53] \\
\hline 68 & 10.58 & $\mathrm{C}_{56} \mathrm{H}_{92} \mathrm{O}_{25}$ & 1164.5932 & 1164.5928 & 0.3 & 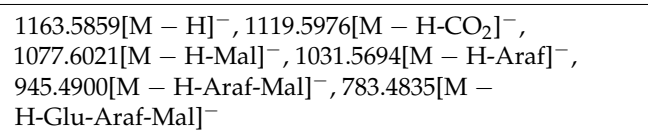 & Malonyl-ginsenoside Rc & WsAG, FgAG & [53] \\
\hline 69 & 10.62 & $\mathrm{C}_{48} \mathrm{H}_{76} \mathrm{O}_{19}$ & 956.4976 & 956.4981 & -0.5 & 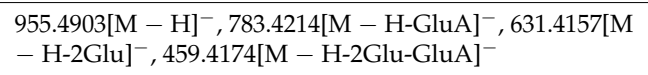 & Ginsenoside Ro & WsAG, FgAG & $\mathrm{s}$ \\
\hline $70^{\#}$ & 10.69 & $\mathrm{C}_{30} \mathrm{H}_{46} \mathrm{O}_{2}$ & 438.3486 & 438.3498 & -2.7 & 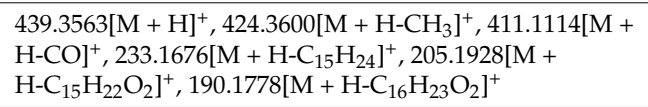 & 3,11-dioxo- $\beta$-amyrene & $\begin{array}{l}\text { WsAG }<\text { FgAG } \\
\text { VIP: } 6.49 \\
p<0.001\end{array}$ & [57] \\
\hline 71 & 10.70 & $\mathrm{C}_{53} \mathrm{H}_{84} \mathrm{O}_{23}$ & 1088.5402 & 1088.5403 & -0.1 & 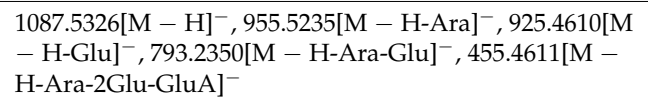 & Stipuleanoside R2 & WsAG, FgAG & [39] \\
\hline 72 & 10.78 & $\mathrm{C}_{53} \mathrm{H}_{90} \mathrm{O}_{22}$ & 1078.5924 & 1078.5924 & 0.0 & 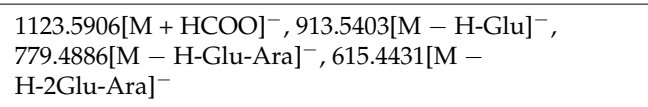 & Ginsenoside $\mathrm{Rb}_{2}$ & WsAG, FgAG & $\mathrm{s}$ \\
\hline 73 & 10.79 & $\mathrm{C}_{53} \mathrm{H}_{90} \mathrm{O}_{22}$ & 1078.5924 & 1078.5924 & 0.0 & 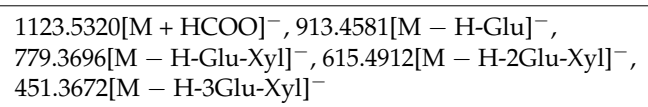 & Ginsenoside $\mathrm{Rb}_{3}$ & WsAG, FgAG & $\mathrm{s}$ \\
\hline 74 & 10.89 & $\mathrm{C}_{55} \mathrm{H}_{92} \mathrm{O}_{23}$ & 1120.6009 & 1120.6029 & -1.8 & 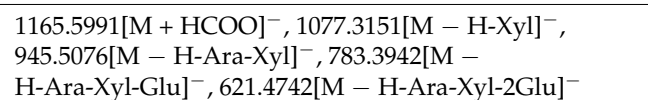 & Notoginsenoside Fc & WsAG, FgAG & [53] \\
\hline 75 & 10.94 & $\mathrm{C}_{56} \mathrm{H}_{92} \mathrm{O}_{25}$ & 1164.5937 & 1164.5928 & 0.8 & $\begin{array}{l}\text { 1163.5864[M - H] }{ }^{-}, 1077.5570\left[\mathrm{M}-\mathrm{H}^{-M a l}\right]^{-} \\
\text {945.5302[M - H-Ara-Mal] } \\
\text { H-Glu-Ara-Mal] }^{-}, \text {, } 621.453 .4540[\mathrm{M}- \\
\text { - }\end{array}$ & Malonyl-ginsenoside $\mathrm{Rb}_{2}$ & WsAG, FgAG & [53] \\
\hline 76 & 11.01 & $\mathrm{C}_{56} \mathrm{H}_{94} \mathrm{O}_{24}$ & 1150.6138 & 1150.6135 & 0.3 & $\begin{array}{l}\text { 1195.6120[M + HCOO }]^{-}, 1149.6060\left[\mathrm{M}-\mathrm{H}^{-}\right. \\
\text {1107.4997[M - H-Ac }]^{-}, 987.4976\left[\mathrm{M}-\mathrm{H}^{-G l u}\right]^{-} \\
\text {945.6047[M - H-Glu-Ac] }{ }^{-}, 783.4864\left[\mathrm{M}-\mathrm{H}-2 \mathrm{Glu}^{-A c}\right]^{-}\end{array}$ & Quinquenoside $\mathrm{R}_{1}$ & WsAG, FgAG & [53] \\
\hline 77 & 11.02 & $\mathrm{C}_{47} \mathrm{H}_{74} \mathrm{O}_{18}$ & 926.4864 & 926.4875 & -1.2 & $\begin{array}{l}\text { 925.4791[M - H }]^{-}, 793.4272\left[\mathrm{M}-\mathrm{H}^{-A r a}\right]^{-}, 612.3784[\mathrm{M} \\
\text { - H-GluA-Ara] }{ }^{-}, 540.3784\left[\mathrm{M}-\mathrm{H}-\mathrm{H} \text {-Glu-C }{ }_{14} \mathrm{H}_{21} \mathrm{O}\right]^{-} \\
\text {455.2841[M - }\end{array}$ & Chikusetsu saponin IV & WsAG, FgAG & [39] \\
\hline
\end{tabular}


Table 1. Cont.

\begin{tabular}{|c|c|c|c|c|c|c|c|c|c|}
\hline No. & $t_{R}(\min )$ & Formula & $\begin{array}{l}\text { Calculated } \\
\text { Mass (Da) }\end{array}$ & $\begin{array}{l}\text { TheoreticalMass } \\
\text { (Da) }\end{array}$ & $\begin{array}{l}\text { Mass Error } \\
(\mathrm{ppm})\end{array}$ & $\mathrm{MS}^{\mathrm{E}}$ Fragmentation & Identification & Sources & Ref. \\
\hline 78 & 11.14 & $\mathrm{C}_{56} \mathrm{H}_{92} \mathrm{O}_{25}$ & 1164.4967 & 1164.4958 & 0.8 & $\begin{array}{l}\text { 1163.4925[M - H] }]^{-}, 1077.5760\left[\mathrm{M}-\mathrm{H}_{-M a l}\right]^{-} \\
\text {945.5503[M - H-Mal-Xyl] } \\
\text { H-Mal-Xyl-Glu] }^{-}, \text {, } 783.4735[\mathrm{M}- \\
\end{array}$ & Malonyl-ginsenoside $\mathrm{Rb}_{3}$ & WsAG, FgAG & [53] \\
\hline 79 & 11.16 & $\mathrm{C}_{36} \mathrm{H}_{62} \mathrm{O}_{9}$ & 638.4395 & 638.4394 & 0.2 & $\begin{array}{l}\text { 683.4366[M + HCOO }]^{-}, 637.4317\left[\mathrm{M}-\mathrm{H}^{-}, 475.2574[\mathrm{M}\right. \\
\left.-\mathrm{H}_{-} \mathrm{Glu}\right]^{-}, 457.2147\left[\mathrm{M}-\mathrm{H}_{-}-\mathrm{Glu}^{-} \mathrm{H}_{2} \mathrm{O}\right]^{-}\end{array}$ & 20(R)-ginsenoside $\mathrm{Rh}_{1}$ & WsAG, FgAG & $\mathrm{s}$ \\
\hline 80 & 11.18 & $\mathrm{C}_{43} \mathrm{H}_{72} \mathrm{O}_{15}$ & 828.4864 & 828.4871 & -0.8 & 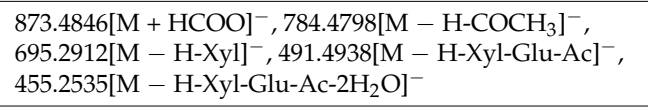 & Vinaginsenoside $\mathrm{R}_{2}$ & WsAG, FgAG & [39] \\
\hline $81^{\#}$ & 11.20 & $\mathrm{C}_{48} \mathrm{H}_{82} \mathrm{O}_{17}$ & 930.5546 & 930.5552 & -0.7 & $\begin{array}{l}\text { 929.5474[M - H }]^{-}, 767.4642[\mathrm{M}-\mathrm{H}-\mathrm{Glu}]^{-}, 605.4365[\mathrm{M} \\
\left.-\mathrm{H}^{-2} \mathrm{Glu}\right]^{-}, 443.1196[\mathrm{M}-\mathrm{H}-3 \mathrm{Glu}]^{-}\end{array}$ & Vinaginsenosides $R_{3}$ & $\begin{array}{c}\text { WsAG }<\text { FgAG } \\
\text { VIP: } 7.60 \\
p<0.001\end{array}$ & {$[58,59]$} \\
\hline 82 & 11.34 & $\mathrm{C}_{42} \mathrm{H}_{66} \mathrm{O}_{14}$ & 794.4447 & 794.4453 & -0.8 & $\begin{array}{l}\text { 793.4368[M - H] }{ }^{-}, 631.3279[\mathrm{M}-\mathrm{H}-\mathrm{Glu}]^{-}, 613.4222[\mathrm{M} \\
\left.-\mathrm{H}-\mathrm{Glu}-\mathrm{H}_{2} \mathrm{O}\right]^{-}, 569.2927\left[\mathrm{M}-\mathrm{H}^{-G l u}-\mathrm{HCOOH}\right]^{-} \\
\text {455.1562[M - H-Glu-GluA }]^{-}\end{array}$ & Chikusetsu saponin II & WsAG, FgAG & [39] \\
\hline 83 & 11.36 & $\mathrm{C}_{48} \mathrm{H}_{82} \mathrm{O}_{18}$ & 946.5508 & 946.5501 & 0.7 & $\begin{array}{l}\text { 991.5490[M + HCOO }]^{-}, 945.5430[\mathrm{M}-\mathrm{H}]^{-}, 783.5147[\mathrm{M} \\
\left.-\mathrm{H}_{-} \mathrm{Glu}\right]^{-}, 459.3241[\mathrm{M}-\mathrm{H}-3 \mathrm{Glu}]^{-}\end{array}$ & Ginsenoside Rd & WsAG, FgAG & $\mathrm{s}$ \\
\hline 84 & 11.39 & $\mathrm{C}_{55} \mathrm{H}_{92} \mathrm{O}_{23}$ & 1120.6029 & 1120.6049 & 1.7 & 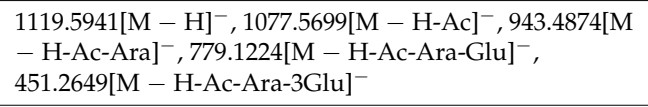 & Ginsenoside $\mathrm{Rs}_{1}$ & WsAG, FgAG & $\mathrm{s}$ \\
\hline 85 & 11.52 & $\mathrm{C}_{51} \mathrm{H}_{84} \mathrm{O}_{21}$ & 1032.5503 & 1032.5505 & -0.2 & $\begin{array}{l}\text { 1031.5425[M - H] }{ }^{-}, 987.5520\left[\mathrm{M}-\mathrm{H}_{-} \mathrm{CO}_{2}\right]^{-}, 945.5192[\mathrm{M} \\
-\mathrm{H}-m a l]^{-}, 783.3540[\mathrm{M}-\mathrm{H}-\mathrm{mal}-\mathrm{Glu}]^{-}, 621.2570[\mathrm{M}- \\
\text { H-mal-2Glu }]^{-}, 459.3458\left[\mathrm{M}-\mathrm{H}^{-m a l-3 G l u}\right]^{-}\end{array}$ & Malonyl-ginsenoside Rd & WsAG, FgAG & [53] \\
\hline 86 & 11.70 & $\mathrm{C}_{55} \mathrm{H}_{92} \mathrm{O}_{23}$ & 1120.6039 & 1120.6029 & 0.8 & $\begin{array}{l}\text { 1165.6021[M + HCOO }]^{-}, 1119.5961\left[\mathrm{M}-\mathrm{H}^{-},\right. \\
\text {987.3684[M - H-Ara] }{ }^{-}, 914.4587[\mathrm{M}-\mathrm{H}-\mathrm{Glu}-\mathrm{Ac}]^{-} \\
\text {458.5471[M - H-3Glu-Ara-Ac] }\end{array}$ & Ginsenoside $\mathrm{Rs}_{2}$ & WsAG, FgAG & $\mathrm{s}$ \\
\hline 87 & 11.88 & $\mathrm{C}_{48} \mathrm{H}_{82} \mathrm{O}_{18}$ & 946.5501 & 946.5500 & -0.1 & $\begin{array}{l}\text { 991.5482[M + HCOO }]^{-}, 783.4871[\mathrm{M}-\mathrm{H}-\mathrm{Glu}]^{-}, \\
603.4416[\mathrm{M}-\mathrm{H}-2 \mathrm{Glu}]^{-}\end{array}$ & Gypenoside XVII & WsAG, FgAG & $\mathrm{s}$ \\
\hline 88 & 12.20 & $\mathrm{C}_{19} \mathrm{H}_{36} \mathrm{O}_{5}$ & 344.2565 & 344.2563 & 0.6 & $\begin{array}{l}343.2486[\mathrm{M}-\mathrm{H}]^{-}, 329.0232\left[\mathrm{M}-\mathrm{H}-\mathrm{CH}_{3}\right]^{-}, 311.2112[\mathrm{M} \\
\left.-\mathrm{H}-\mathrm{H}_{2} \mathrm{O}-\mathrm{CH}_{3}\right]^{-}, 294.1609\left[\mathrm{M}-\mathrm{H}-\mathrm{H}_{2} \mathrm{O}-\mathrm{OCH}_{3}\right]^{-} \\
255.1494\left[\mathrm{M}-\mathrm{H}_{-} \mathrm{OCH}_{3}-\mathrm{C}_{4} \mathrm{H}_{9}\right]^{-}, 242.1610[\mathrm{M}- \\
\left.\mathrm{H}-\mathrm{OCH}_{3}-\mathrm{C}_{5} \mathrm{H}_{11}\right]^{-}, 228.2523\left[\mathrm{M}-\mathrm{H}-\mathrm{OCH}_{3}-\mathrm{C}_{6} \mathrm{H}_{12}\right]^{-}\end{array}$ & $\begin{array}{l}\text { Methyl-9,10,11-trihydroxy-12- } \\
\text { octadecenoate }\end{array}$ & WsAG, FgAG & [60] \\
\hline 89 & 12.27 & $\mathrm{C}_{47} \mathrm{H}_{80} \mathrm{O}_{17}$ & 916.5398 & 916.5396 & 0.2 & 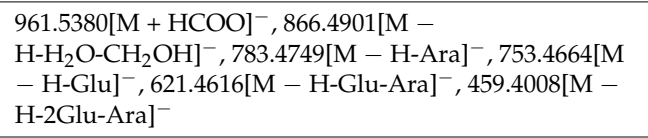 & Notoginsenoside Fe & WsAG, FgAG & [39] \\
\hline 90 & 12.38 & $\mathrm{C}_{50} \mathrm{H}_{84} \mathrm{O}_{19}$ & 988.5594 & 988.5607 & -1.3 & $\begin{array}{l}\text { 1033.5576[M + HCOO }]^{-}, 987.5539\left[\mathrm{M}-\mathrm{H}^{-}, 945.5428[\mathrm{M}\right. \\
\left.-\mathrm{H}-\mathrm{COCH}_{3}\right]^{-}, 809.4326\left[\mathrm{M}-\mathrm{H}-2 \mathrm{H}_{2} \mathrm{O}-\mathrm{C}_{8} \mathrm{H}_{14} \mathrm{O}_{2}\right]^{-} \\
\left.\text {797.4813[M }-\mathrm{H}-\mathrm{Glu}-\mathrm{C}_{2} \mathrm{H}_{4}\right]^{-}\end{array}$ & Quinquenoside III & WsAG, FgAG & [61] \\
\hline
\end{tabular}


Table 1. Cont

\begin{tabular}{|c|c|c|c|c|c|c|c|c|c|}
\hline No. & $t_{R}(\min )$ & Formula & $\begin{array}{l}\text { Calculated } \\
\text { Mass (Da) }\end{array}$ & $\begin{array}{l}\text { TheoreticalMass } \\
\text { (Da) }\end{array}$ & $\begin{array}{l}\text { Mass Error } \\
(\mathrm{ppm})\end{array}$ & $\mathrm{MS}^{\mathrm{E}}$ Fragmentation & Identification & Sources & Ref. \\
\hline $91^{\#}$ & 12.48 & $\mathrm{C}_{47} \mathrm{H}_{80} \mathrm{O}_{17}$ & 916.5385 & 916.5396 & -1.1 & 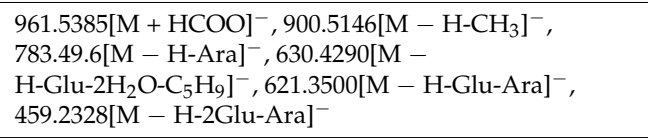 & Chikusetsu saponin III & $\begin{array}{l}\text { WsAG }<\text { FgAG } \\
\text { VIP: } 12.78 \\
p<0.001\end{array}$ & [39] \\
\hline 92 & 12.57 & $\mathrm{C}_{30} \mathrm{H}_{46} \mathrm{O}_{2}$ & 766.4855 & 766.4867 & -1.6 & $\begin{array}{l}\text { 767.4928[M + H }{ }^{+}, 749.3674\left[\mathrm{M}+\mathrm{H}_{-} \mathrm{H}_{2} \mathrm{O}\right]^{+}, 621.2398[\mathrm{M}+ \\
\left.\mathrm{H}^{-R h a}\right]^{+}, 459.2280[\mathrm{M}+\mathrm{H}-\mathrm{Glu}-\mathrm{Rha}]^{+}, 207.1780[\mathrm{M}+ \\
\left.\text { H-Glu-Rha- }_{16} \mathrm{H}_{26} \mathrm{O}\right]^{+}\end{array}$ & $(20 E)$-Ginsenoside $\mathrm{F}_{4}$ & WsAG, FgAG & [62] \\
\hline 93 & 12.68 & $\mathrm{C}_{47} \mathrm{H}_{80} \mathrm{O}_{17}$ & 916.5399 & 916.5396 & 0.3 & $\begin{array}{l}\text { 961.5381[M + HCOO] }{ }^{-}, 814.4616\left[\mathrm{M}-\mathrm{H}-\mathrm{H}_{2} \mathrm{O}-\mathrm{C}_{6} \mathrm{H}_{11}\right]^{-} \\
783.4923[\mathrm{M}-\mathrm{H}-\mathrm{Xyl}]^{-}, 621.4390[\mathrm{M}-\mathrm{H}-\mathrm{Hlu}-\mathrm{Xyl}]^{-}\end{array}$ & Gypenoside IX & WsAG, FgAG & [53] \\
\hline 94 & 13.14 & $\mathrm{C}_{42} \mathrm{H}_{70} \mathrm{O}_{14}$ & 798.4748 & 798.4765 & -2.1 & 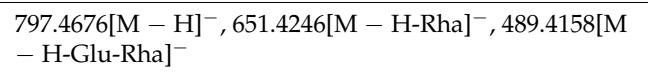 & Ginsenoside $\operatorname{Rg}_{8}$ & WsAG, FgAG & [63] \\
\hline 95 & 13.18 & $\mathrm{C}_{52} \mathrm{H}_{86} \mathrm{O}_{19}$ & 1014.5756 & 1014.5763 & -0.6 & 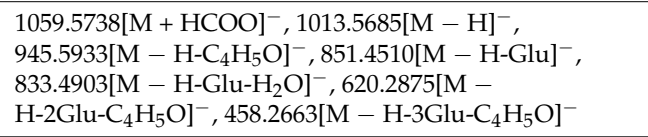 & Quinquenoside I & WsAG, FgAG & [61] \\
\hline 96 & 13.31 & $\mathrm{C}_{48} \mathrm{H}_{82} \mathrm{O}_{17}$ & 930.5548 & 930.5552 & -0.4 & 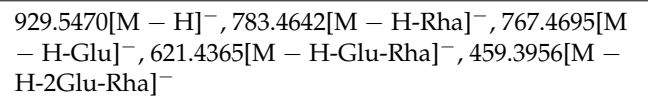 & Gypenoside X & WsAG, FgAG & - \\
\hline 97 & 13.43 & $\mathrm{C}_{42} \mathrm{H}_{70} \mathrm{O}_{12}$ & 766.4861 & 766.4867 & -0.8 & $\begin{array}{l}\text { 765.4783[M + HCOO }]^{-}, 610.2361[\mathrm{M}- \\
\left.\mathrm{H}-\mathrm{CH}_{2} \mathrm{OH}-\mathrm{C}_{8} \mathrm{H}_{13}-\mathrm{CH}_{3}\right]^{-}, 603.2375\left[\mathrm{M}-\mathrm{H}^{-} \mathrm{Glu}^{-}\right. \\
441.1811[\mathrm{M}-\mathrm{H}-2 \mathrm{Glu}]^{-}, 340.2323\left[\mathrm{M}-\mathrm{H}-\mathrm{C}_{30} \mathrm{H}_{49} \mathrm{O}\right]^{-}\end{array}$ & Ginsenoside $\mathrm{Rk}_{1}$ & WsAG, FgAG & [39] \\
\hline 98 & 13.50 & $\mathrm{C}_{47} \mathrm{H}_{74} \mathrm{O}_{18}$ & 926.4862 & 926.4875 & -1.4 & 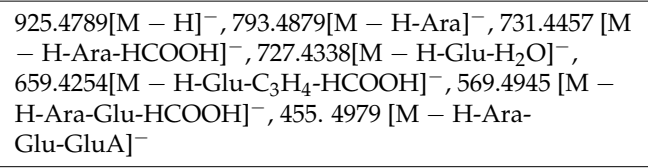 & Chikusetsu saponin Ib & WsAG, FgAG & [39] \\
\hline $99 \#$ & 13.52 & $\mathrm{C}_{42} \mathrm{H}_{72} \mathrm{O}_{13}$ & 784.4974 & 784.4973 & -0.1 & $\begin{array}{l}\text { 783.4896[M - H }]^{-}, 737.4755\left[\mathrm{M}-\mathrm{H}-\mathrm{CH}_{2} \mathrm{OH}-\mathrm{CH}_{3}\right]^{-} \\
660.4330\left[\mathrm{M}-\mathrm{H}-3 \mathrm{H}_{2} \mathrm{O}-\mathrm{C}_{5} \mathrm{H}_{9}\right]^{-}, 621.4361\left[\mathrm{M}-\mathrm{H}^{-G l u}\right]^{-} \\
459.3782\left[\mathrm{M}-\mathrm{H}-2 \mathrm{Glu}^{-}\right.\end{array}$ & Ginsenoside $F_{2}$ & $\begin{array}{l}\text { WsAG }<\text { FgAG } \\
\text { VIP: } 17.01 \\
p<0.001\end{array}$ & [39] \\
\hline 100 & 13.57 & $\mathrm{C}_{18} \mathrm{H}_{30} \mathrm{O}_{4}$ & 310.2141 & 310.2144 & -1.0 & 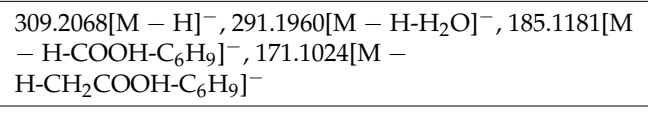 & $\begin{array}{l}\text { 13S-hydroperoxy-9Z,11E,15Z- } \\
\text { octadecatrienoic acid }\end{array}$ & WsAG, FgAG & [64] \\
\hline 101 & 13.62 & $\mathrm{C}_{36} \mathrm{H}_{60} \mathrm{O}_{7}$ & 604.4334 & 604.4339 & -0.8 & $\begin{array}{l}\text { 605.4407[M + H]+ }]^{+}, 586.4285\left[\mathrm{M}+\mathrm{H}_{-} \mathrm{H}_{2} \mathrm{O}\right]^{+}, 443.3860[\mathrm{M}+ \\
\mathrm{H}-\mathrm{Glu}^{+}, 405.3657\left[\mathrm{M}+\mathrm{H}-\mathrm{Glu}-\mathrm{H}_{2} \mathrm{O}\right]^{+}, 333.0939[\mathrm{M}+ \\
\left.\mathrm{H}-2 \mathrm{H}_{2} \mathrm{O}-\mathrm{C}_{16} \mathrm{H}_{26} \mathrm{O}\right]^{+}, 296.1006\left[\mathrm{M}+\mathrm{H}-\mathrm{Glu} \mathrm{H}_{2} \mathrm{O}-\mathrm{C}_{8} \mathrm{H}_{13}\right]^{+}\end{array}$ & Isoginsenoside $\mathrm{Rh}_{3}$ & WsAG, FgAG & [65] \\
\hline $102 *$ & 13.93 & $\mathrm{C}_{42} \mathrm{H}_{66} \mathrm{O}_{14}$ & 794.4449 & 794.4453 & -0.5 & $\begin{array}{l}\text { 793.4387[M - H }]^{-}, 613.3751[\mathrm{M}-\mathrm{H}-\mathrm{Glu}]^{-}, 569.3830[\mathrm{M} \\
\left.-\mathrm{H}-\mathrm{Glu}^{-} \mathrm{H}_{2} \mathrm{O}-\mathrm{CO}_{2}\right]^{-}\end{array}$ & Chikusetsusaponin Iva & $\begin{array}{l}\text { WsAG }>\text { FgAG } \\
\text { VIP:16.17 } \\
p<0.001\end{array}$ & [53] \\
\hline
\end{tabular}


Table 1. Cont.

\begin{tabular}{|c|c|c|c|c|c|c|c|c|c|}
\hline No. & $t_{R}(\min )$ & Formula & $\begin{array}{l}\text { Calculated } \\
\text { Mass (Da) }\end{array}$ & $\begin{array}{l}\text { TheoreticalMass } \\
\text { (Da) }\end{array}$ & $\begin{array}{l}\text { Mass Error } \\
(\mathrm{ppm})\end{array}$ & $\mathrm{MS}^{\mathrm{E}}$ Fragmentation & Identification & Sources & Ref. \\
\hline 103 & 14.51 & $\mathrm{C}_{42} \mathrm{H}_{72} \mathrm{O}_{13}$ & 784.4967 & 784.4973 & -0.7 & $\begin{array}{l}\text { 829.4951[M + HCOO }]^{-}, 783.4892[\mathrm{M}-\mathrm{H}]^{-}, 621.4442[\mathrm{M} \\
\left.-\mathrm{H}_{-} \mathrm{Glu}\right]^{-}, 459.3684[\mathrm{M}-\mathrm{H}-2 \mathrm{Glu}]^{-}\end{array}$ & 20(R)-Ginsenoside $\mathrm{Rg}_{3}$ & WsAG, FgAG & $\mathrm{s}$ \\
\hline 104 & 14.64 & $\mathrm{C}_{17} \mathrm{H}_{30} \mathrm{O}_{2}$ & 266.2246 & 266.2246 & -0.1 & $\begin{array}{l}\text { 311.2228[M + HCOO }]^{-}, 168.1023\left[\mathrm{M}-\mathrm{H}_{-} \mathrm{C}_{7} \mathrm{H}_{13}\right]^{-}, \\
154.1074\left[\mathrm{M}-\mathrm{H}^{-} \mathrm{C}_{8} \mathrm{H}_{15}\right]^{-}, 137.2250[\mathrm{M}- \\
\left.\mathrm{H}-\mathrm{C}_{7} \mathrm{H}_{13}-\mathrm{OCH}_{3}\right]^{-}, 115.0352\left[\mathrm{M}-\mathrm{H}-\mathrm{C}_{4} \mathrm{H}_{7}-\mathrm{C}_{6} \mathrm{H}_{9} \mathrm{O}\right]^{-}, \\
96.0352\left[\mathrm{M}-\mathrm{H}_{-} \mathrm{C}_{11} \mathrm{H}_{21} \mathrm{O}\right]^{-}\end{array}$ & $\begin{array}{l}\text { 5-Hexenoic acid, 10-undecenyl } \\
\text { ester }\end{array}$ & WsAG, FgAG & a \\
\hline $105^{\#}$ & 14.74 & $\mathrm{C}_{18} \mathrm{H}_{28} \mathrm{O}_{2}$ & 276.2081 & 276.2089 & -2.9 & 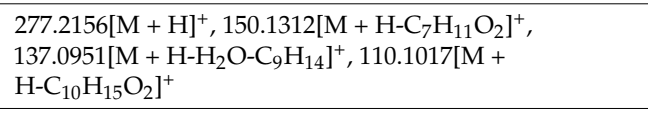 & Palmitoleic acid & $\begin{array}{c}\text { WsAG }<\text { FgAG } \\
\text { VIP: } 8.57 \\
p<0.001\end{array}$ & $\mathrm{~s}$ \\
\hline 106 & 14.75 & $\mathrm{C}_{42} \mathrm{H}_{72} \mathrm{O}_{13}$ & 784.4940 & 784.4973 & -4.1 & $\begin{array}{l}\text { 829.4951[M + HCOO }]^{-}, 783.4865[\mathrm{M}-\mathrm{H}]^{-}, 621.4942[\mathrm{M} \\
-\mathrm{H}-\mathrm{Glu}]^{-}, 459.4578[\mathrm{M}-\mathrm{H}-2 \mathrm{Glu}]^{-}, 441.5214[\mathrm{M}- \\
\left.\mathrm{H}-2 \mathrm{Glu}-\mathrm{H}_{2} \mathrm{O}\right]^{-}\end{array}$ & 20(S)-Ginsenoside $\operatorname{Rg}_{3}$ & WsAG, FgAG & $\mathrm{s}$ \\
\hline 107 & 14.90 & $\mathrm{C}_{41} \mathrm{H}_{70} \mathrm{O}_{12}$ & 754.4874 & 754.4867 & 0.8 & $\begin{array}{l}\text { 799.4856[M + HCOO }]^{-}, 621.3141[\mathrm{M}-\mathrm{H}-\mathrm{Xyl}]^{-}, \\
459.3887\left[\mathrm{M}-\mathrm{H}-\mathrm{Glu}^{-}-\mathrm{Xyl}\right]^{-}, 351.2556[\mathrm{M}- \\
\left.\mathrm{H}-\mathrm{Xyl}-\mathrm{H}_{2} \mathrm{O}-\mathrm{C}_{16} \mathrm{H}_{28} \mathrm{O}_{2}\right]^{-}, 275.1442[\mathrm{M}- \\
\left.\text { H-Glu-Xyl-2C }{ }_{6} \mathrm{H}_{11}\right]^{-}\end{array}$ & Gypenoside XIII & WsAG, FgAG & [66] \\
\hline 108 & 14.98 & $\mathrm{C}_{41} \mathrm{H}_{64} \mathrm{O}_{13}$ & 764.4345 & 764.4347 & -0.3 & 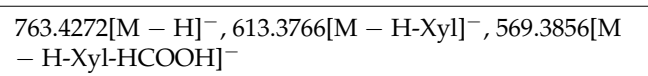 & Pseudo-ginsenoside $\mathrm{Rp}_{1}$ & WsAG, FgAG & [39] \\
\hline 109 & 15.05 & $\mathrm{C}_{17} \mathrm{H}_{30} \mathrm{O}_{2}$ & 266.2244 & 266.2246 & -0.7 & $\begin{array}{l}\text { 311.2226[M + } \mathrm{HCOO}^{-}, 222.1128\left[\mathrm{M}-\mathrm{H}_{-} \mathrm{C}_{3} \mathrm{H}_{7}\right]^{-} \\
139.0826\left[\mathrm{M}-\mathrm{H}_{-} \mathrm{C}_{9} \mathrm{H}_{19}\right]^{-}, 127.1127\left[\mathrm{M}-\mathrm{H}_{-} \mathrm{C}_{8} \mathrm{H}_{11} \mathrm{O}_{2}\right]^{-}\end{array}$ & (2E,4E)-Hydroprene & WsAG, FgAG & $\mathrm{a}$ \\
\hline 110 & 15.75 & $\mathrm{C}_{43} \mathrm{H}_{68} \mathrm{O}_{14}$ & 808.4610 & 808.4609 & 0.1 & 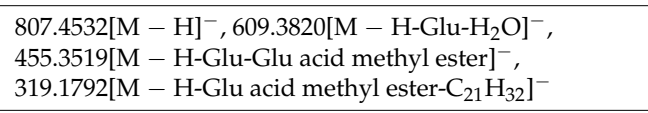 & $\begin{array}{l}\text { Chikusetsusaponin IVa methyl } \\
\text { ester }\end{array}$ & WsAG, FgAG & [39] \\
\hline 111 & 15.98 & $\mathrm{C}_{18} \mathrm{H}_{30} \mathrm{O}_{3}$ & 294.2194 & 294.2195 & -0.3 & $\begin{array}{l}293.2122[\mathrm{M}-\mathrm{H}]^{-}, 275.2013\left[\mathrm{M}-\mathrm{H}_{-} \mathrm{H}_{2} \mathrm{O}\right]^{-}, \\
\left.-\mathrm{H}_{-} \mathrm{C}_{9} \mathrm{H}_{15}\right]^{-}, 171.1024[\mathrm{M} \\
\end{array}$ & $\begin{array}{l}(E, E) \text {-9-Oxooctadeca-10,12-dienoic } \\
\text { acid }\end{array}$ & WsAG, FgAG & [34] \\
\hline $112 *$ & 16.90 & $\mathrm{C}_{36} \mathrm{H}_{62} \mathrm{O}_{8}$ & 622.4431 & 622.4445 & -2.1 & $\begin{array}{l}\text { 667.4442[M + HCOO }]^{-}, 621.4360\left[\mathrm{M}-\mathrm{H}^{-}, 459.2656[\mathrm{M}\right. \\
-\mathrm{H}-\mathrm{Glu}]^{-}, 441.4772\left[\mathrm{M}-\mathrm{H}-\mathrm{Glu}^{-} \mathrm{H}_{2} \mathrm{O}\right]^{-}\end{array}$ & Ginsenoside $\mathrm{Rh}_{2}$ & $\begin{array}{c}\text { WsAG > FgAG } \\
\text { VIP: } 4.68 \\
p=0.0032\end{array}$ & $\mathrm{~s}$ \\
\hline 113 & 17.34 & $\mathrm{C}_{18} \mathrm{H}_{32} \mathrm{O}_{3}$ & 296.2347 & 296.2351 & 1.4 & $\begin{array}{l}\text { 295.2274[M - H }]^{-}, 278.2172\left[\mathrm{M}-\mathrm{H}_{-} \mathrm{H}_{2} \mathrm{O}\right]^{-}, 233.2273[\mathrm{M} \\
-\mathrm{H}-\mathrm{HCOOH}-]^{-}, 184.1182\left[\mathrm{M}-\mathrm{H}-\mathrm{C}_{8} \mathrm{H}_{15}\right]^{-} \\
\left.\text {171.1023[M }-\mathrm{H}^{-} \mathrm{C}_{9} \mathrm{H}_{16}\right]^{-}, 148.1125\left[\mathrm{M}-\mathrm{H}_{-} \mathrm{C}_{8} \mathrm{H}_{15} \mathrm{O}_{2}\right]^{-} \\
\left.\text {125.1174[M }-\mathrm{H}-\mathrm{H}_{2} \mathrm{O}-\mathrm{C}_{10} \mathrm{H}_{17} \mathrm{O}\right]^{-}\end{array}$ & $\begin{array}{l}\text { 9-Hydroxyoctadeca-10,12-dienoic } \\
\text { acid }\end{array}$ & WsAG, FgAG & [67] \\
\hline 114 & 17.37 & $\mathrm{C}_{42} \mathrm{H}_{70} \mathrm{O}_{12}$ & 766.4860 & 766.4867 & -0.9 & $\begin{array}{l}\text { 811.4933[M + } \mathrm{HCOO}^{-}, 747.4834\left[\mathrm{M}-\mathrm{H}^{-} \mathrm{H}_{2} \mathrm{O}^{-}\right. \\
603.4833\left[\mathrm{M}-\mathrm{H}^{-G l u}\right]^{-}, 585.4309\left[\mathrm{M}-\mathrm{H}^{-G l u}\right]^{-} \\
459.0768\left[\mathrm{M}-\mathrm{H}_{-}-\mathrm{Glu}-\mathrm{Rha}\right]^{-}, 421.4457\left[\mathrm{M}-\mathrm{H}^{-G l u}-\mathrm{Rha}\right]^{-}\end{array}$ & Ginsenoside $\operatorname{Rg}_{5}$ & WsAG, FgAG & [53] \\
\hline $115^{\#}$ & 17.45 & $\mathrm{C}_{18} \mathrm{H}_{30} \mathrm{O}_{2}$ & 278.2245 & 278.2246 & -0.1 & $\begin{array}{l}\text { 279.2321[M + H }]^{+}, 218.1936\left[\mathrm{M}+\mathrm{H}-\mathrm{HCOOH}-\mathrm{CH}_{3}\right]^{+} \\
\left.\text {184.1479[M }+\mathrm{H}-\mathrm{C}_{7} \mathrm{H}_{11}\right]^{+}\end{array}$ & $\alpha$-Linolenic Acid & $\begin{array}{c}\text { WsAG }<\text { FgAG } \\
\text { VIP: } 5.24 \\
p<0.001\end{array}$ & $\mathrm{~s}$ \\
\hline
\end{tabular}


Table 1. Cont

\begin{tabular}{|c|c|c|c|c|c|c|c|c|c|}
\hline No. & $t_{R}(\min )$ & Formula & $\begin{array}{l}\text { Calculated } \\
\text { Mass (Da) }\end{array}$ & $\begin{array}{l}\text { TheoreticalMass } \\
\text { (Da) }\end{array}$ & $\begin{array}{l}\text { Mass Error } \\
(\text { ppm) }\end{array}$ & $\mathrm{MS}^{\mathrm{E}}$ Fragmentation & Identification & Sources & Ref. \\
\hline 116 & 18.24 & $\mathrm{C}_{32} \mathrm{H}_{50} \mathrm{O}_{4}$ & 498.3722 & 498.3709 & 2.4 & $\begin{array}{l}\text { 521.3614[M+Na }]^{+}, 484.3365\left[\mathrm{M}+\mathrm{H}-\mathrm{CH}_{3}\right]^{+}, 439.3322[\mathrm{M}+ \\
\left.\mathrm{H}-\mathrm{C}_{2} \mathrm{H}_{3} \mathrm{O}_{2}\right]^{+}, 303.3080\left[\mathrm{M}-\mathrm{C}_{12} \mathrm{H}_{20} \mathrm{O}_{2}\right]^{+} \\
263.2783\left[\mathrm{M}-\mathrm{C}_{15} \mathrm{H}_{24} \mathrm{O}_{2}\right]^{+}, 248.2610\left[\mathrm{M}+\mathrm{H}-\mathrm{C}_{16} \mathrm{H}_{26} \mathrm{O}_{2}\right]^{+} \\
\left.\text {203.0991[M }+\mathrm{H}^{-} \mathrm{C}_{2} \mathrm{H}_{3} \mathrm{O}_{2}-\mathrm{C}_{15} \mathrm{H}_{22} \mathrm{O}_{2}\right]^{+}\end{array}$ & 3-O-Acetyloleanolic acid & WsAG, FgAG & a \\
\hline $117^{*}$ & 18.50 & $\mathrm{C}_{19} \mathrm{H}_{24} \mathrm{O}_{2}$ & 284.1773 & 284.1776 & -1.1 & $\begin{array}{l}285.1843[\mathrm{M}+\mathrm{H}]^{+}, 259.2243\left[\mathrm{M}+\mathrm{H}-\mathrm{C}_{2} \mathrm{H}_{2}\right]^{+}, 243.1701[\mathrm{M}+ \\
\left.\mathrm{H}-\mathrm{C}_{2} \mathrm{H}_{2} \mathrm{O}\right]^{+}, 159.1308\left[\mathrm{M}+\mathrm{H}-\mathrm{C}_{8} \mathrm{H}_{13} \mathrm{O}\right]^{+}, 122.1168[\mathrm{M}+ \\
\left.\mathrm{H}-\mathrm{C}_{11} \mathrm{H}_{11} \mathrm{O}\right]^{+}\end{array}$ & Androsta-1,4-diene-3,17-dione & $\begin{array}{l}\text { WsAG }>\text { FgAG } \\
\text { VIP: } 6.16 \\
p<0.001\end{array}$ & a \\
\hline 118 & 19.97 & $\mathrm{C}_{16} \mathrm{H}_{28} \mathrm{O}_{3}$ & 268.2039 & 268.2038 & 0.4 & 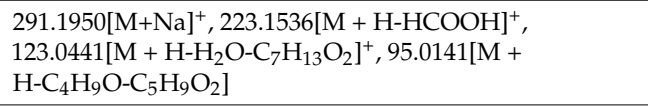 & $\begin{array}{l}\text { 13-Hydroxy-9,11-hexadecanedioic } \\
\text { acid }\end{array}$ & WsAG, FgAG & [34] \\
\hline 119 & 20.15 & $\mathrm{C}_{17} \mathrm{H}_{24} \mathrm{O}_{2}$ & 260.1766 & 260.1776 & -3.8 & $\begin{array}{l}261.1839[\mathrm{M}+\mathrm{H}]^{+}, 243.1708\left[\mathrm{M}+\mathrm{H}-\mathrm{H}_{2} \mathrm{O}\right]^{+}, 221.1479[\mathrm{M}+ \\
\left.\mathrm{H}-\mathrm{CH}_{3}-\mathrm{C}_{2} \mathrm{H}_{3}\right]^{+}, 159.0791\left[\mathrm{M}+\mathrm{H}-\mathrm{H}_{2} \mathrm{O}-\mathrm{C}_{6} \mathrm{H}_{13}\right]^{+}\end{array}$ & Panaxydol & WsAG, FgAG & {$[68]$} \\
\hline 120 & 20.49 & $\mathrm{C}_{17} \mathrm{H}_{26} \mathrm{O}_{3}$ & 280.3138 & 280.3130 & 3.3 & 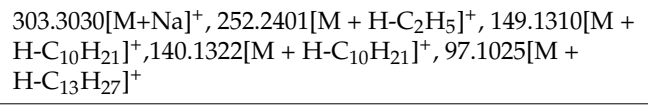 & 1-Eicosene & WsAG, FgAG & a \\
\hline 121 & 22.88 & $\mathrm{C}_{19} \mathrm{H}_{38} \mathrm{O}_{4}$ & 330.2766 & 330.2770 & -1.2 & $\begin{array}{l}353.2658[\mathrm{M}+\mathrm{Na}]^{+}, 313.2725\left[\mathrm{M}+\mathrm{H}-\mathrm{H}_{2} \mathrm{O}\right]^{+}, 280.2603[\mathrm{M}+ \\
\left.\mathrm{H}-2 \mathrm{H}_{2} \mathrm{O}-\mathrm{CH}_{3}\right]^{+}, 239.2352\left[\mathrm{M}+\mathrm{H}-\mathrm{C}_{3} \mathrm{H}_{7} \mathrm{O}_{3}\right]^{+}, 99.0871[\mathrm{M}+ \\
\left.\mathrm{H}^{-} \mathrm{C}_{4} \mathrm{H}_{7} \mathrm{O}_{4}-\mathrm{C}_{8} \mathrm{H}_{17}\right]^{+}\end{array}$ & Monopalmitin & WsAG, FgAG & {$[30]$} \\
\hline
\end{tabular}

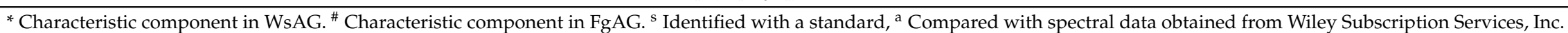
(New York, NY, USA). 

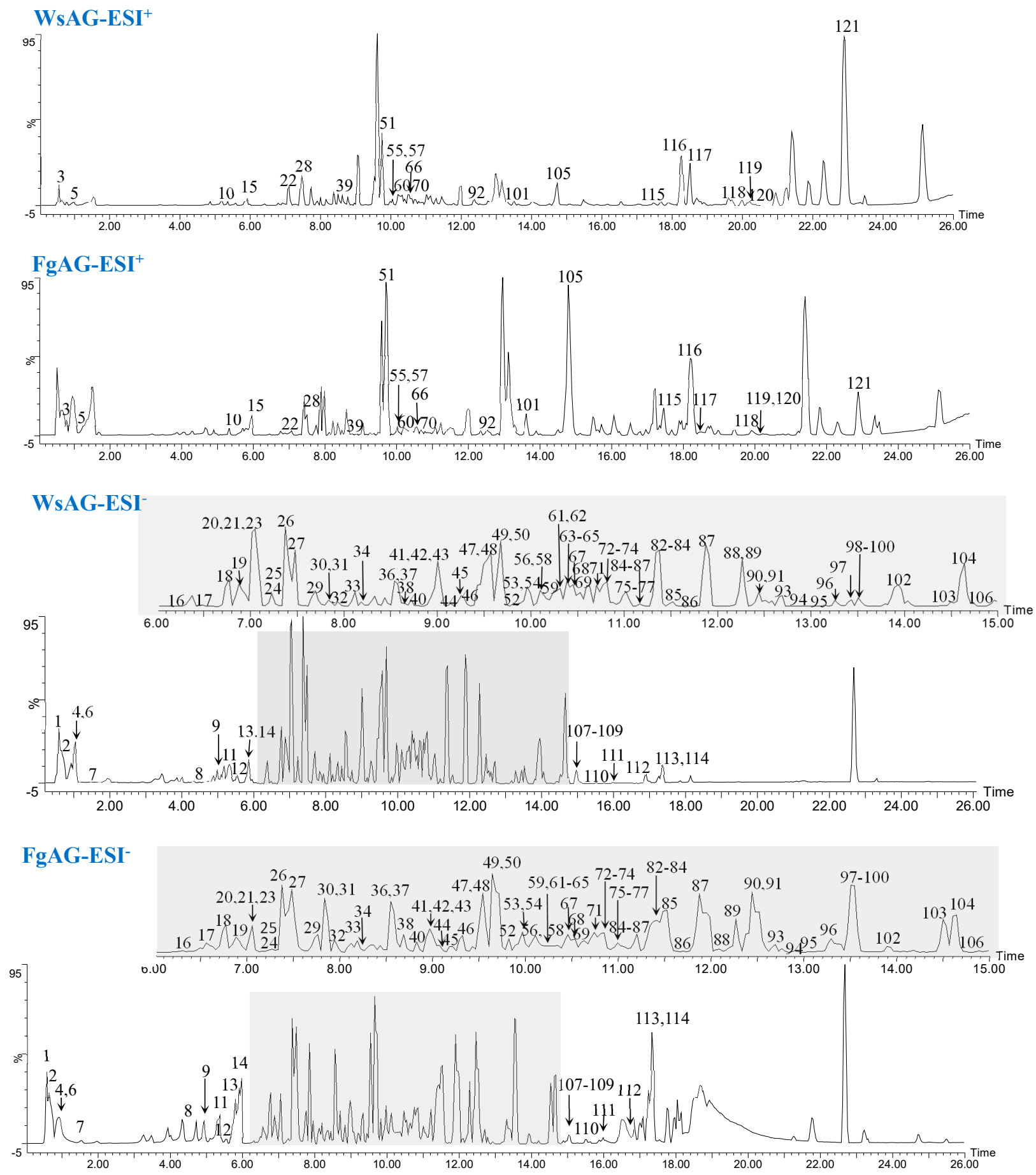

Figure 1. The representative base peak intensity (BPI) chromatograms of FgAG and WsAG in negative and positive modes. 
Triterpenoids

Dammarane ginsenosides

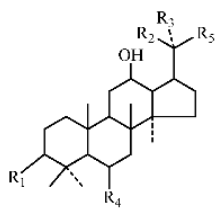<smiles>CCCCC(C)CC(C)CO</smiles><smiles>CC(C)CCCCC(C)C(C)C</smiles><smiles>CC(C)=CC=C(C)C</smiles><smiles>CCC(C)(C)C(C)O</smiles><smiles>CCCC(C)(C)O</smiles><smiles>CC=CC(O)C(C)C</smiles><smiles>[Y9]C1CCC2C(CC(O)C3C(C)CCC23)C1CC(C)C</smiles><smiles>CC(C)=CCCC(C)(C)C</smiles><smiles>CCCCC</smiles>

$\mathrm{J}$

Protopanaxtriol-type saponins

13. $\mathrm{R}_{1}-\mathrm{OH}, \mathrm{R}_{2}-\mathrm{OGlu}, \mathrm{R}_{3}-\mathrm{CH}_{3}, \mathrm{R}_{4}-\mathrm{OGlu}^{4}-\mathrm{I}_{-}^{\mathrm{G}} \mathrm{Glu}, \mathrm{R}_{5}-\mathrm{A}$ 17. $\mathrm{R}_{1}=\mathrm{OH}, \mathrm{R}_{2}=\mathrm{OGlu}{ }^{6}{ }_{-}^{1} \mathrm{Glu}, \mathrm{R}_{3}-\mathrm{CH}_{3}, \mathrm{R}_{4}-\mathrm{OGlu}^{2}{ }_{-}{ }^{\mathrm{R}} \mathrm{R} a, \mathrm{R}_{5}-\mathrm{A}$ 18. $\mathrm{R}_{1}-\mathrm{OII}, \mathrm{R}_{2}-\mathrm{OGlu}^{6}{ }_{-}{ }^{\prime} \mathrm{Araf}, \mathrm{R}_{3}-\mathrm{CII}_{3}, \mathrm{R}_{4}-\mathrm{OGlu}, \mathrm{R}_{5}-\mathrm{A}$ 21. $\mathrm{R}_{1}=\mathrm{OH}, \mathrm{R}_{2}=\mathrm{OGlu}, \mathrm{R}_{3}=\mathrm{CH}_{3}, \mathrm{R}_{4}=\mathrm{OGlu}{ }^{2}{ }_{-} \mathrm{Xyl}, \mathrm{R}_{5}=\mathrm{A}$ 23. $\mathrm{R}_{1}=\mathrm{OH}, \mathrm{R}_{2}=\mathrm{OGlu}^{6}{ }^{-1} \mathrm{Glu}, \mathrm{R}_{3}=\mathrm{CH}_{3}, \mathrm{R}_{4}=\mathrm{OGlu}, \mathrm{R}_{5}=\mathrm{A}$ $26 \mathrm{R}_{1}=\mathrm{OH}, \mathrm{R}_{2}=\mathrm{OGlu}, \mathrm{R}_{3}=\mathrm{CH}_{3}, \mathrm{R}_{4}=\mathrm{OGlu}, \mathrm{R}_{5}=\mathrm{A}$

$27 \mathrm{R}_{1}-$ OHI, $\mathrm{R}_{2}$-OGlu, $\mathrm{R}_{3}-\mathrm{CH}_{3}, \mathrm{R}_{4}-\mathrm{OGlu}^{2}-\mathrm{I}^{\mathrm{N}} \mathrm{Rha}, \mathrm{R}_{5}-\mathrm{A}$ $29 \mathrm{R}_{1}=\mathrm{OH}, \mathrm{R}_{2}=\mathrm{OGlu}, \mathrm{R}_{3}=\mathrm{CH}_{3}, \mathrm{R}_{4}=\mathrm{OGlu}^{6}-\mathrm{Mal}, \mathrm{R}_{5}=\mathrm{A}$ 31. $\mathrm{R}_{1}-\mathrm{OH}, \mathrm{R}_{2}-\mathrm{OGlu}, \mathrm{R}_{3}-\mathrm{CH}_{3}, \mathrm{R}_{4}-\mathrm{OMal}^{6}{ }^{6} \mathrm{Glu}^{2}-{ }^{\prime} \mathrm{Rlla}, \mathrm{R}_{5}-\mathrm{A}$ 36. $\mathrm{R}_{1}-\mathrm{OII}, \mathrm{R}_{2}-\mathrm{OGlu}^{6}-\mathrm{Ac}, \mathrm{R}_{3}-\mathrm{CII}_{3}, \mathrm{R}_{4}-\mathrm{OGlu} \mathrm{R}_{5}-\mathrm{A}$

37. $\mathrm{R}_{1}=\mathrm{OH}, \mathrm{R}_{2}=\mathrm{OH}, \mathrm{R}_{3}=\mathrm{CH}_{3}, \mathrm{R}_{4}=\mathrm{OGlu}^{2}-{ }^{\prime} \mathrm{Arap}, \mathrm{R}_{5}=\mathrm{A}$

Protopanaxdiol-type saponins

33. $\mathrm{R}_{1}=\mathrm{OGlu}^{2}-{ }^{\prime} \mathrm{Glu}, \mathrm{R}_{2}=\mathrm{O}-\mathrm{Ara}, \mathrm{R}_{3}=\mathrm{CH}_{3}, \mathrm{R}_{4}=\mathrm{H}, \mathrm{R}_{5}=\mathrm{A}$

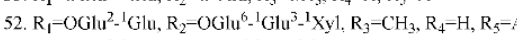

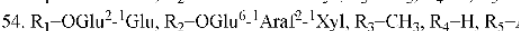

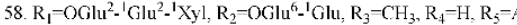
59. $\mathrm{R}_{1}=\mathrm{OGlu}^{2}{ }_{-1} \mathrm{Gllu} \mathrm{R}_{2}=\mathrm{OGllu}^{6}{ }^{6}{ }^{\mathrm{l}} \mathrm{Glu}, \mathrm{R}_{3}=\mathrm{CH}_{3}, \mathrm{R}_{4}=\mathrm{H}, \mathrm{R}_{5}=\mathrm{A}$

62. $\mathrm{R}_{1}-\mathrm{OGlu}^{2}-\mathrm{I}^{\prime} \mathrm{Glu}^{6}-\mathrm{Mal}, \mathrm{R}_{2}-\mathrm{OGlu}^{6}-\mathrm{I}^{\prime} \mathrm{Glu}, \mathrm{R}_{3}-\mathrm{CII}_{3}, \mathrm{R}_{4}-\mathrm{II}, \mathrm{R}_{5}-\mathrm{A}$ 65. $\mathrm{R}_{1}=\mathrm{OGlu}^{2}{ }_{-1}^{-1} \mathrm{Glu}, \mathrm{R}_{2}=\mathrm{OGilu}^{6}{ }_{-1}{ }_{-1} \mathrm{Araf}, \mathrm{R}_{3}=\mathrm{CH}_{3}, \mathrm{R}_{4}=\mathrm{H}, \mathrm{R}_{5}=\mathrm{A}$

67. $\mathrm{R}_{1}-\mathrm{OGlu}^{2}{ }^{1}{ }^{1} \mathrm{Glu}, \mathrm{R}_{2}=\mathrm{OGlu}^{6}{ }_{-}{ }^{-} \mathrm{Arap}^{4}-{ }^{1} \mathrm{Xyl}, \mathrm{R}_{3}-\mathrm{CH}_{3}, \mathrm{R}_{4}-\mathrm{II}, \mathrm{R}_{5}-\mathrm{A}$ 68. $\mathrm{R}_{1}-\mathrm{OGlu}^{2}{ }_{-} \mathrm{GGl}^{6}-\mathrm{Mal}, \mathrm{R}_{2}-\mathrm{OGlu}^{6}-\mathrm{A}$ Ara, $\mathrm{R}_{3}-\mathrm{CII}_{3}, \mathrm{R}_{4}-\mathrm{II}_{1}, \mathrm{R}_{5}-\mathrm{A}$ 72. $\mathrm{R}_{1}=\mathrm{OGlu}^{2}{ }^{-1} \mathrm{Glu}, \mathrm{R}_{2}=\mathrm{OGlu}^{6}{ }_{-1}{ }_{\mathrm{Arap}}, \mathrm{R}_{3}=\mathrm{CH}_{3}, \mathrm{R}_{4}=\mathrm{H}_{4} \mathrm{R}_{5}=\mathrm{A}$ 73. $\mathrm{R}_{1}-\mathrm{OGlu}^{2}-\mathrm{I}^{-} \mathrm{Glu}, \mathrm{R}_{2}-\mathrm{OGlu}^{6}-\mathrm{XXl}^{-} \mathrm{R}_{3}-\mathrm{CH}_{3}, \mathrm{R}_{4}-\mathrm{H}, \mathrm{R}_{5}-\mathrm{A}$ 74. $\mathrm{R}_{1}=\mathrm{OGlu}^{2}{ }_{-} \mathrm{Gllu}^{6}-\mathrm{Ac}, \mathrm{R}_{2}=\mathrm{OGlu}^{6}-\mathrm{Arap} \mathrm{R}_{3}=\mathrm{CH}_{3}, \mathrm{R}_{4}=\mathrm{H}, \mathrm{R}_{5}=\mathrm{A}$ 75. $\mathrm{R}_{1}-\mathrm{OGlu}^{2}{ }_{-} \mathrm{GGl}^{6}-\mathrm{Mal}, \mathrm{R}_{2}-\mathrm{OGlu}^{6}-\mathrm{I}^{-}$Arap, $\mathrm{R}_{3}-\mathrm{CII}_{3}, \mathrm{R}_{4}-\mathrm{II}, \mathrm{R}_{5}-\mathrm{A}$ 76. $\mathrm{R}_{1}=\mathrm{OGlu}^{2}-{ }^{1} \mathrm{Glu}^{6}-\mathrm{Ac}, \mathrm{R}_{2}=\mathrm{OGlu}^{6}-{ }^{1} \mathrm{Glu}, \mathrm{R}_{3}=\mathrm{CH}_{3}, \mathrm{R}_{4}=\mathrm{H}, \mathrm{R}_{5}=\mathrm{A}$ 78. $\mathrm{R}_{1}-\mathrm{OGlu}^{2}-\mathrm{C}^{-1} \mathrm{Gl}^{6}-\mathrm{Mal}, \mathrm{R}_{2}-\mathrm{OGlu}^{6}-{ }^{6} \mathrm{Xyl}_{1}, \mathrm{R}_{3}-\mathrm{CH}_{3}, \mathrm{R}_{4}-\mathrm{H}, \mathrm{R}_{5}-\mathrm{A}$ 81. $\mathrm{R}_{1}-$ OGlu'- Glu, $\mathrm{R}_{2}-\mathrm{OGlu}, \mathrm{R}_{3}-\mathrm{CH}_{3}, \mathrm{R}_{4}-\mathrm{H}, \mathrm{R}_{5}-\mathrm{A}$ 83. $\mathrm{R}_{1}=\mathrm{OGlu}^{2}{ }_{-}^{\mathrm{I}} \mathrm{Glu}, \mathrm{R}_{2}=\mathrm{OGlu}, \mathrm{R}_{3}=\mathrm{CH}_{3}, \mathrm{R}_{4}=\mathrm{H}, \mathrm{R}_{5}=\mathrm{A}$ 84. $\mathrm{R}_{1}-\mathrm{OGlu}^{2}{ }^{-1} \mathrm{Gll}_{1}-\mathrm{Ac}, \mathrm{R}_{2}-\mathrm{OGlu}^{6}{ }^{6}{ }^{1} \mathrm{Arap}, \mathrm{R}_{3}-\mathrm{CH}_{3}, \mathrm{R}_{4}-\mathrm{H}, \mathrm{R}_{5}-\mathrm{A}$ 85. $\mathrm{R}_{1}=\mathrm{OGlu}^{2}{ }^{-1}$ Glu-Mal, $\mathrm{R}_{2}=\mathrm{OGlu}, \mathrm{R}_{3}=\mathrm{CH}_{3}, \mathrm{R}_{4}=\mathrm{H}, \mathrm{R}_{5}=\mathrm{A}$

86. $\mathrm{R}_{1}=\mathrm{OGlu}^{2}{ }_{-1}^{1} \mathrm{Glu}^{6}-\mathrm{Ac}, \mathrm{R}_{2}=\mathrm{OGlu}{ }^{6}-\mathrm{A}^{\mathrm{A} a f}, \mathrm{R}_{3}=\mathrm{CH}_{3}, \mathrm{R}_{4}=\mathrm{H}, \mathrm{R}_{5}=\mathrm{A}$ 87. $\mathrm{R}_{1}-$ OGlu, $\mathrm{R}_{2}-\mathrm{OGlu}^{6}{ }_{-}{ }^{-} \mathrm{Glu}, \mathrm{R}_{3}-\mathrm{CII}_{3}, \mathrm{R}_{4}-\mathrm{II}$. $\mathrm{R}_{5}-\mathrm{A}$

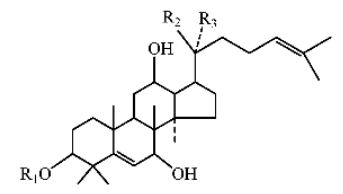

25. $\mathrm{R}_{1}-\mathrm{Gl}_{\mathrm{G}}{ }^{6}-\mathrm{I}_{2} \mathrm{Glu}, \mathrm{R}_{2}-\mathrm{Glu}^{6}{ }_{-}{ }^{1} \mathrm{Glu}, \mathrm{R}_{3}-\mathrm{CH}_{3}$ 41. $\mathrm{R}_{1}-\mathrm{Gl}^{2}-\mathrm{I}^{-} \mathrm{Glu}, \mathrm{R}_{2}-\mathrm{Glu}^{6}-\mathrm{X} \mathrm{Xyl}, \mathrm{R}_{3}-\mathrm{CH}_{3}$ 48. $\mathrm{R}_{1}=\mathrm{Glu}^{2}{ }_{-}^{1} \mathrm{Glu}, \mathrm{R}_{2}=\mathrm{Glu}, \mathrm{R}_{3}=\mathrm{CH}_{3}$
53. $\mathrm{R}_{1}=\mathrm{OH}, \mathrm{R}_{2}=\mathrm{OGilu}^{2}-{ }^{\prime}$ Araf, $\mathrm{R}_{3}-\mathrm{CH}_{3}, \mathrm{R}_{4}=\mathrm{OH}, \mathrm{R}_{5}=\mathrm{A}$ 61. $\mathrm{R}_{1}-\mathrm{OH}, \mathrm{R}_{2}-\mathrm{CH}_{3}, \mathrm{R}_{3}-\mathrm{OH}, \mathrm{R}_{4}-\mathrm{OGlu}{ }^{2}{ }_{-1} \mathrm{Rha} . \mathrm{R}_{5}-\mathrm{A}$ 63. $\mathrm{R}_{1}=\mathrm{OH}, \mathrm{R}_{2}=\mathrm{OH}, \mathrm{R}_{3}=\mathrm{CH}_{3}, \mathrm{R}_{4}=\mathrm{OGlu}, \mathrm{R}_{5}=\mathrm{A}$ 64. $\mathrm{R}_{1}=\mathrm{OH}, \mathrm{R}_{2}=\mathrm{OGlu}{ }^{2}{ }_{-}{ }^{\mathrm{A}}$ arap, $\mathrm{R}_{3}=\mathrm{CH}_{3}, \mathrm{R}_{4}=\mathrm{OH}, \mathrm{R}_{5}=\mathrm{A}$ 79. $\mathrm{R}_{1}=\mathrm{OH}, \mathrm{R}_{2}=\mathrm{CH} 3, \mathrm{R}_{3}=\mathrm{OH}, \mathrm{R}_{4}=\mathrm{OGlu}, \mathrm{R}_{5}=\mathrm{A}$ 9. $\mathrm{R}_{1}-\mathrm{OII}, \mathrm{R}_{2}-\mathrm{OGlu}, \mathrm{R}_{3}-\mathrm{CII}_{3}, \mathrm{R}_{4}-\mathrm{OGlu}^{2}{ }^{-} \mathrm{Rha}_{1} \mathrm{R}_{5}-\mathrm{G}$ 14. $\mathrm{R}_{1}=\mathrm{OH}, \mathrm{R}_{2}=\mathrm{OH}, \mathrm{R}_{3}=\mathrm{CH}_{3}, \mathrm{R}_{4}=\mathrm{OGlu}^{2}{ }^{1} \mathrm{R}$ Ra. $\mathrm{R}_{5}=\mathrm{I}$ 30. $\mathrm{R}_{1}=\mathrm{OH}, \mathrm{R}_{2}=\mathrm{OH}, \mathrm{R}_{3}=\mathrm{CH}_{3}, \mathrm{R}_{4}=\mathrm{OGlu}^{2}{ }_{-}{ }^{\mathrm{R}} \mathrm{Rh}, \mathrm{R}_{5}=\mathrm{E}$ 38. $\mathrm{R}_{1}-\mathrm{OII}, \mathrm{R}_{2}-\mathrm{OGlu}_{2} \mathrm{R}_{3}-\mathrm{Cl}_{3}, \mathrm{R}_{4}-\mathrm{OGlu}^{2}-\mathrm{Rha}^{\mathrm{R}} \mathrm{R}_{5}-\mathrm{I}$ 66. $\mathrm{R}_{1}=\mathrm{OH}, \mathrm{R}_{4}=$ OGilu, $\mathrm{R}_{6}=\mathrm{K}$

92. $\mathrm{R}_{1}-\mathrm{OH}, \mathrm{R}_{4}-\mathrm{OGlu}^{2}-{ }^{-1} \mathrm{Rha}, \mathrm{R}_{6}-\mathrm{L}$

94. $\mathrm{R}_{1}-\mathrm{OH}, \mathrm{R}_{4}-\mathrm{OGlu}^{2}-{ }^{-}$Rha, $\mathrm{R}_{6}-\mathrm{M}$

89. $\mathrm{R}_{1}=$ OGlu, $\mathrm{R}_{2}=\mathrm{OGilu}^{6}{ }_{-1}{ }_{\mathrm{Araf},} \mathrm{R}_{3}=\mathrm{CH}_{3}, \mathrm{R}_{4}=\mathrm{H}, \mathrm{R}_{5}=\mathrm{A}$ 90. $\mathrm{R}_{1}=\mathrm{OGlu}^{2}-\mathrm{I}^{1} \mathrm{Glu}^{6}$-Actyl, $\mathrm{R}_{2}=\mathrm{OGlu}, \mathrm{R}_{3}=\mathrm{CH}_{3}, \mathrm{R}_{4}=\mathrm{H}, \mathrm{R}_{5}=\mathrm{A}$ 91. $\mathrm{R}_{1}=\mathrm{OArap}{ }_{-}{ }^{6} \mathrm{Gllu}^{2}{ }_{-}{ }_{\mathrm{Glu}} \mathrm{R}_{2}=\mathrm{OH}, \mathrm{R}_{3}=\mathrm{CH}_{3}, \mathrm{R}_{4}=\mathrm{H}, \mathrm{R}_{5}=\mathrm{A}$ 93. $\mathrm{R}_{1}=\mathrm{OG} \mid \mathrm{lu}, \mathrm{R}_{2}=\mathrm{OGlu}{ }^{6}-1 \mathrm{Xyl}, \mathrm{R}_{3}=\mathrm{CH}_{3}, \mathrm{R}_{4}=\mathrm{H}, \mathrm{R}_{5}=\mathrm{A}$

95. $\mathrm{R}_{1}=\mathrm{OGIl}^{2}{ }^{2} \mathrm{Gilu}^{6}$-1 -oxo-2-butenyl, $\mathrm{R}_{2}=\mathrm{OGlu}, \mathrm{R}_{3}=\mathrm{CH}_{3}, \mathrm{R}_{4}=\mathrm{H}, \mathrm{R}_{5}=\mathrm{\Lambda}$ 96. $\mathrm{R}_{1}=\mathrm{OG} \mid \mathrm{u}, \mathrm{R}_{2}=\mathrm{OGGlu}_{-}{ }_{-1} \mathrm{Rha}_{3} \mathrm{R}_{3}=\mathrm{CH}_{3}, \mathrm{R}_{4}=\mathrm{H}, \mathrm{R}_{5}=\Lambda$ 99. $\mathrm{R}_{1}-$ OGlu, $\mathrm{R}_{2}-$ OGlu, $\mathrm{R}_{3}-\mathrm{CII}_{3}, \mathrm{R}_{4}-\mathrm{II}, \mathrm{R}_{5}-\mathrm{A}$

103. $\mathrm{R}_{1}=\mathrm{OGlu}^{2}{ }^{-1} \mathrm{Glu}, \mathrm{R}_{2}=\mathrm{CH}_{3}, \mathrm{R}_{3}=\mathrm{OH}, \mathrm{R}_{4}=\mathrm{H}, \mathrm{R}_{3}=\mathrm{A}$ 106. $\mathrm{R}_{1}=\mathrm{OGlu}^{2}{ }_{-1}^{\mathrm{I}} \mathrm{Glu}, \mathrm{R}_{2}=\mathrm{OH}, \mathrm{R}_{3}=\mathrm{CH}_{3}, \mathrm{R}_{4}=\mathrm{H}, \mathrm{R}_{5}=\mathrm{A}$ 107. $\mathrm{R}_{1}=\mathrm{OH}, \mathrm{R}_{2}=\mathrm{OGlu}^{2}{ }^{1} \mathrm{Xyl}, \mathrm{R}_{3}=\mathrm{CH}_{3}, \mathrm{R}_{4}=\mathrm{H}, \mathrm{R}_{5}=\mathrm{A}$ 112. $\mathrm{R}_{1}-\mathrm{OGlu}^{2}, \mathrm{R}_{2}-\mathrm{OH}, \mathrm{R}_{3}-\mathrm{CH}_{3}, \mathrm{R}_{4}-\mathrm{H}, \mathrm{R}_{5}-\mathrm{A}$

15. $\mathrm{R}_{1}=$ Glu, $\mathrm{R}_{2}=-\mathrm{OGlu}, \mathrm{R}_{3}=\mathrm{CH}_{3}, \mathrm{R}_{4}=\mathrm{H}, \mathrm{R}_{5}=\mathrm{B}$

43. $\mathrm{R}_{1}=\mathrm{OGlu}^{2}{ }_{-} \mathrm{I}_{\mathrm{Glu}}, \mathrm{R}_{2}=-\mathrm{OGlu}, \mathrm{R}_{3}=\mathrm{CH}_{3}, \mathrm{R}_{4}=\mathrm{H}, \mathrm{R}_{5}=\mathrm{B}$ 44. $\mathrm{R}_{1}=\mathrm{OGlu}^{2}{ }_{-} \mathrm{IGlu}_{2} \mathrm{R}_{2}=\mathrm{OH}, \mathrm{R}_{3}=\mathrm{CH}_{3}, \mathrm{R}_{4}=\mathrm{H}, \mathrm{R}_{5}=\mathrm{C}$ 35. $\mathrm{R}_{1}=\mathrm{OGlu}^{2}{ }_{-}{ }^{\mathrm{G}} \mathrm{Glu}, \mathrm{R}_{2}=\mathrm{OGlu}^{6}{ }_{-}{ }_{\mathrm{Xyl}} \mathrm{XY}, \mathrm{R}_{3}=\mathrm{CH}_{3}, \mathrm{R}_{4}=\mathrm{H}, \mathrm{R}_{3}=\mathrm{D}$ 19. $\mathrm{R}_{1}=\mathrm{OGlu}^{2} \mathrm{I}_{\mathrm{G}} \mathrm{Glu}, \mathrm{R}_{2}=\mathrm{OGilu}, \mathrm{R}_{3}=\mathrm{CH}_{3}, \mathrm{R}_{4}=\mathrm{H}, \mathrm{R}_{5}=\mathrm{E}$ 40. $\mathrm{R}_{1}-\mathrm{OGlu}^{2}{ }_{-}{ }^{\mathrm{I}} \mathrm{Glu}, \mathrm{R}_{2}-\mathrm{OGlu}, \mathrm{R}_{3}-\mathrm{CH}_{3}, \mathrm{R}_{4}-\mathrm{H}, \mathrm{R}_{5}-\mathrm{F}$ 42. $\mathrm{R}_{1}-\mathrm{OGlu}, \mathrm{R}_{2}-\mathrm{OGlu}^{2}{ }_{-1} \mathrm{Xyl}_{1} \mathrm{R}_{3}-\mathrm{CH}_{3}, \mathrm{R}_{4}-\mathrm{H}_{4} \mathrm{R}_{5}-\mathrm{F}$ 24. $\mathrm{R}_{1}-\mathrm{OGll}^{2}-\mathrm{I}^{-} \mathrm{Glu} \mathrm{u}_{-}{ }^{-} \mathrm{Xyl}, \mathrm{R}_{2}-\mathrm{OH}, \mathrm{R}_{3}-\mathrm{CH}_{3} \mathrm{R}_{4}-\mathrm{H}, \mathrm{R}_{5}-\mathrm{O}$

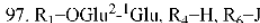
101. $\mathrm{R}_{1}=$ OGilu, $\mathrm{R}_{4}=\mathrm{H}, \mathrm{R}_{6}=\mathrm{K} \quad$ 114. $\mathrm{R}_{1}-\mathrm{OGlu}^{2}-\mathrm{I}_{\mathrm{G}}$ Gilu, $\mathrm{R}_{4}-\mathrm{H}, \mathrm{R}_{6}-\mathrm{K}$

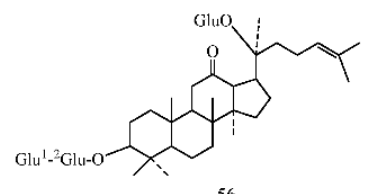

Figure 2. Cont.
Ocotillol-type saponins

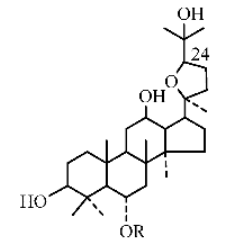

1. $\mathrm{R}=\mathrm{OGlu}^{1}{ }_{-}^{6} \mathrm{Glu}^{2}-{ }^{1} \mathrm{Glu} \quad 24 R$ 12. $\mathrm{R}=\mathrm{OGlu}^{2}{ }_{-}^{1} \mathrm{Glu}^{2}{ }_{-}^{-1} \mathrm{Ara} \quad 24 R$

20. $\mathrm{R}=\mathrm{OGilu}^{2}-{ }^{-1} \mathrm{Rha}$

32. $\mathrm{R}=\mathrm{Glu}^{1}-{ }^{6} \mathrm{OGl}{ }^{2}-{ }^{-1} \mathrm{Xyl} \quad 24$.

34. $\mathrm{R}=\mathrm{Ac}-{ }^{6} \mathrm{OGlu}^{2}-{ }^{-1} \mathrm{Rha} \quad 24 \mathrm{~S}$

47. $\mathrm{R}=\mathrm{OGlu}{ }^{2}-{ }^{1} \mathrm{Xyl}$

49. $\mathrm{R}=\mathrm{OGlu}^{2}-{ }^{-} \mathrm{Rh}$

50. $\mathrm{R}=\mathrm{OGlu}$

51. $\mathrm{R}=\mathrm{OGlu}$

Oleanane-type saponins

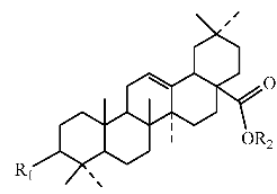

10. $\mathrm{R}_{1}=\mathrm{OHI}, \mathrm{R}_{2}=\mathrm{Gilu}$

39. $\mathrm{R}_{1}=\mathrm{H}, \mathrm{R}_{2}=\mathrm{H}$

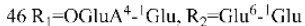

55. $\mathrm{R}_{1}=\mathrm{OArap}^{2}{ }^{-1} \mathrm{Rha}, \mathrm{R}_{2}=\mathrm{II}$

69. $\mathrm{R}_{1}-\mathrm{OGlu}_{\mathrm{A}} \mathrm{N}^{2} \mathrm{-} \mathrm{Glu}, \mathrm{R}_{2}-\mathrm{Glu}$

71. $\mathrm{R}_{1}=\mathrm{OGlu}^{1}{ }_{-}^{3} \mathrm{OGluA}^{4}{ }_{-} \mathrm{Araf}^{\mathrm{A}} \mathrm{R}_{2}=\mathrm{Glu}$

77. $\mathrm{R}_{1}-\mathrm{OGlu} \Lambda^{4}-{ }^{-1} \Lambda \mathrm{raf}, \mathrm{R}_{2}-\mathrm{Glu}$

82. $\mathrm{R}_{1}-\mathrm{OGlu}^{-} \mathrm{N}^{6}{ }^{1} \mathrm{Glu}, \mathrm{R}_{2}-\mathrm{H}$

98. $\mathrm{R}_{1}-\mathrm{O}$ - $\mathrm{raf}^{1}-{ }_{-}^{4} \mathrm{Glu} \Lambda^{6}-{ }_{-}^{\mathrm{I}} \mathrm{Glu}, \mathrm{R}_{2}-\mathrm{II}$

102. $R_{1}=$ OGluA, $R_{2}=$ Glu

108. $\mathrm{R}_{1}=\mathrm{OGluA}^{2}-\mathrm{Xyl}^{1}, \mathrm{R}_{2}=\mathrm{I}$

110. $\mathrm{R}_{1}$-OGluA-methyl ester, $\mathrm{R}_{2}-\mathrm{Gl}$

116. $\mathrm{R}_{1}=\mathrm{OCOCH}_{3} \mathrm{R}_{2}=\mathrm{H}$
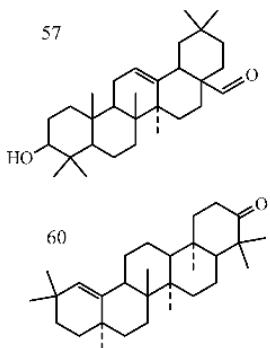

Ursane

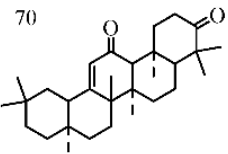




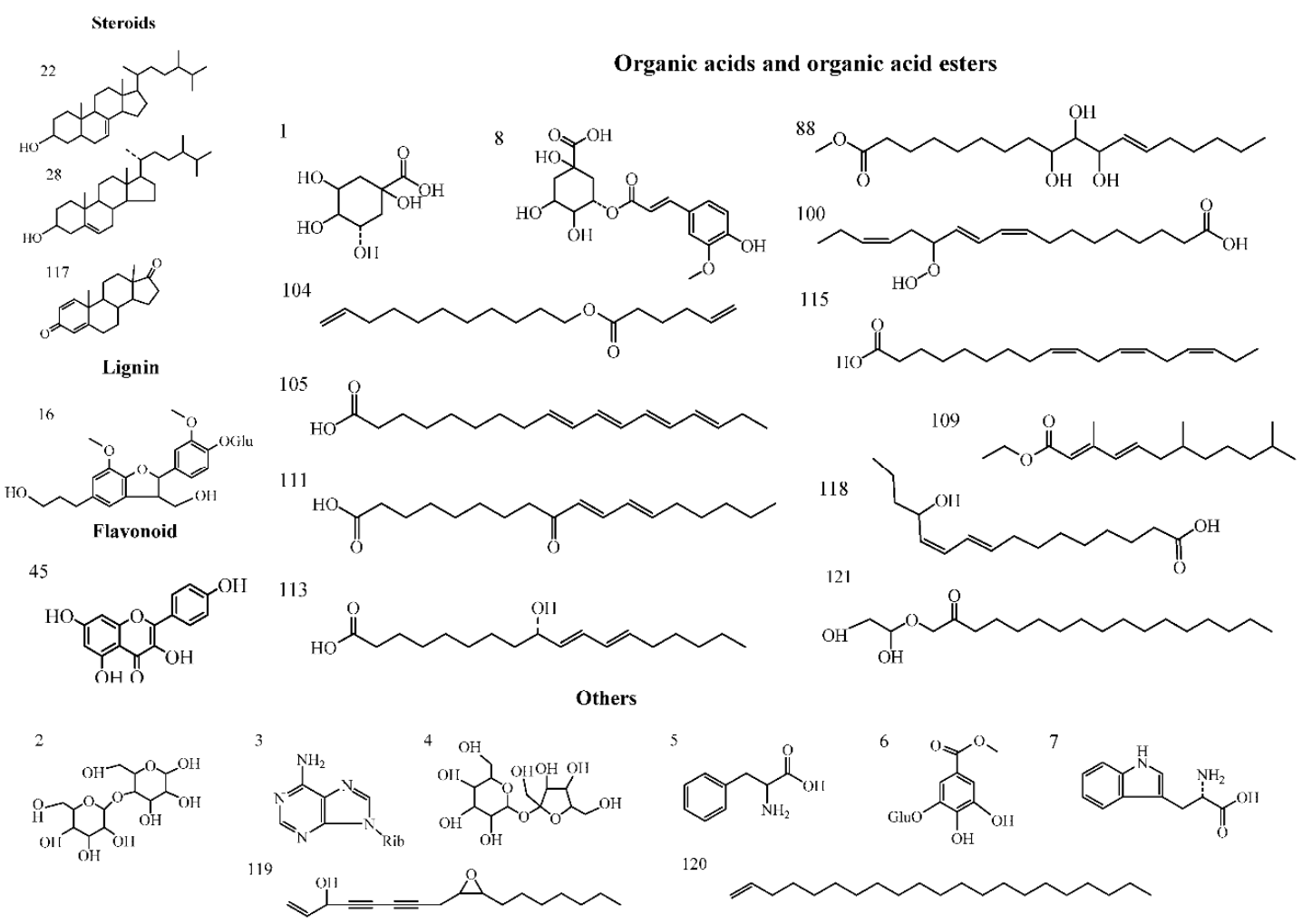

Figure 2. Chemical structures of compounds identified in FgAG and WsAG.

In FgAG and WsAG, these compounds were all shared constituents, including 47 protopanaxdiol-type saponins, 23 protopanaxtriol-type saponins, 15 oleanane- type saponins, 10 ocotillol-type saponins, one ursane, one flavonoid, one lignin, 12 organic acids and organic acid esters, three steroids, and eight other compounds.

\subsection{Biomarker Discovery for $F g A G$ and $W s A G$}

The QC injections were clustered tightly in PCA indicating a satisfactory stability of the system. The PCA 2D plots of the samples from FgAG and WsAG groups were classified into two clusters according to their common spectral characteristics (Figure 3), with the FgAG samples of different years clustered into one group, while the WsAG samples were clustered into another group. The FgAG and WsAG samples were clearly separated, indicating that these two herb species could be easily differentiated.
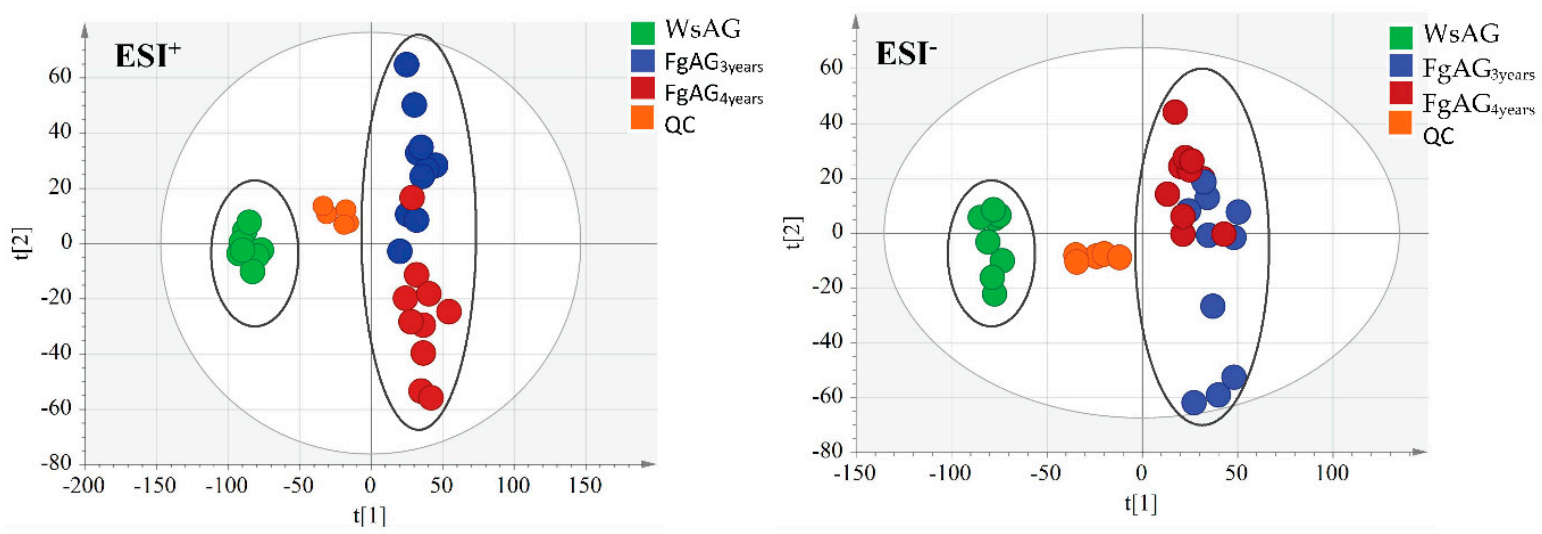

Figure 3. The PCA of FgAG and WsAG in positive mode and negative mode. 
After OPLS-DA plots (Figures 4A and 5A) in both negative and positive modes were generated, the maximum separation between MsAG and FgAG groups was available. In the sufficient permutation test, the lines of grouping samples were significantly located underneath the random sampling lines (Figures $4 \mathrm{~B}$ and $5 \mathrm{~B}$ ), which indicated a fine validity for the following characteristic metabolites biomarkers identification [42]. S-plots were then created to explore the potential chemical markers that contributed to the differences. Based on $p$ values $(p<0.05)$ and VIP values (VIP $>3)[30,61]$ from univariate statistical analysis, 22 robust known biomarkers enabling the differentiation between FgAG and WsAG, were marked and listed (Figures $4 \mathrm{C}$ and $5 \mathrm{C}$ and Table 2). Additionally, a heatmap was generated from these biomarkers in order to systematically evaluate the biomarkers (Figure 6), which visually showed the intensities of potential biomarkers between two species.
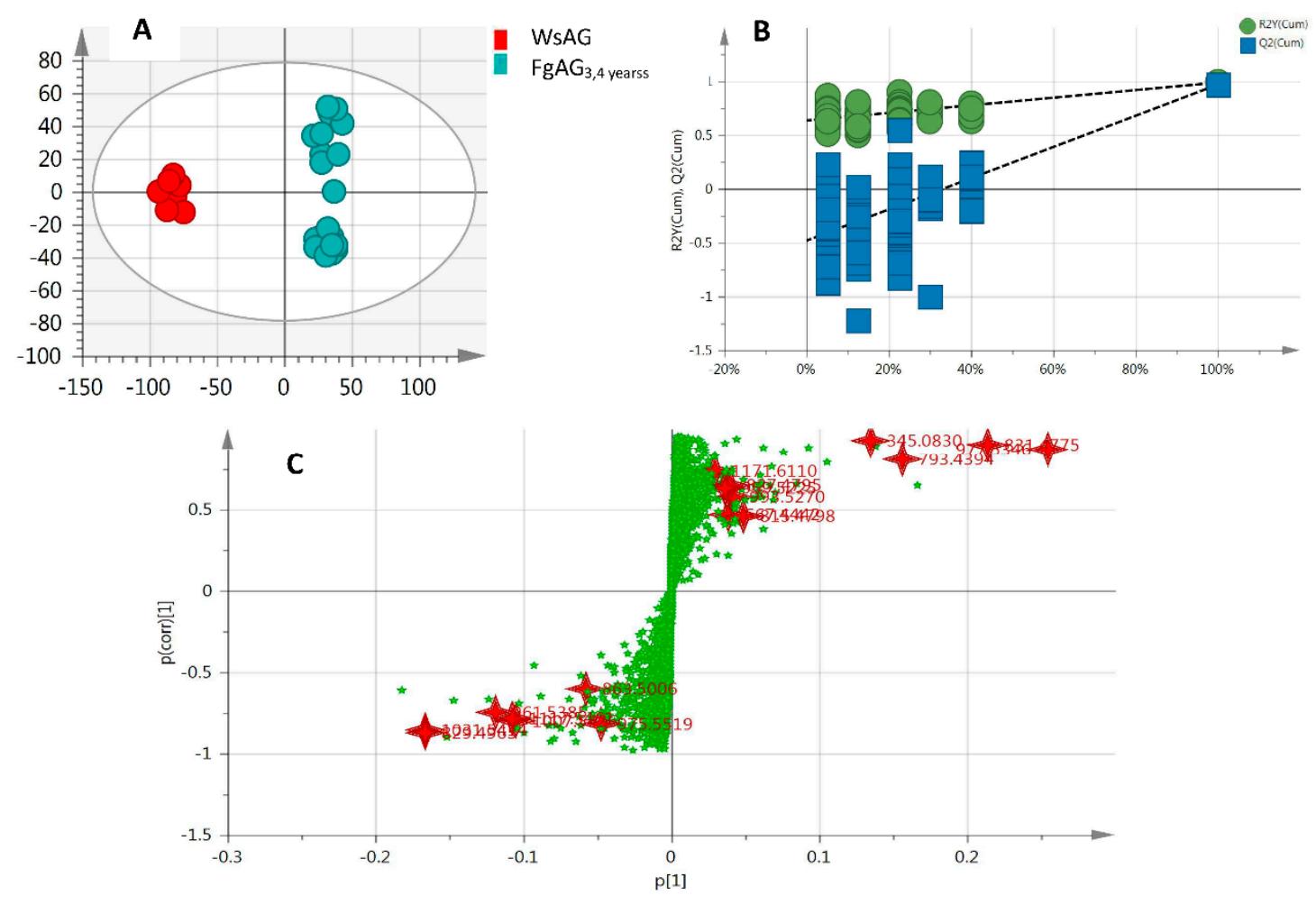

Figure 4. The OPLS-DA (A); permutation tests (B) and S-plot (C) in negative mode. 

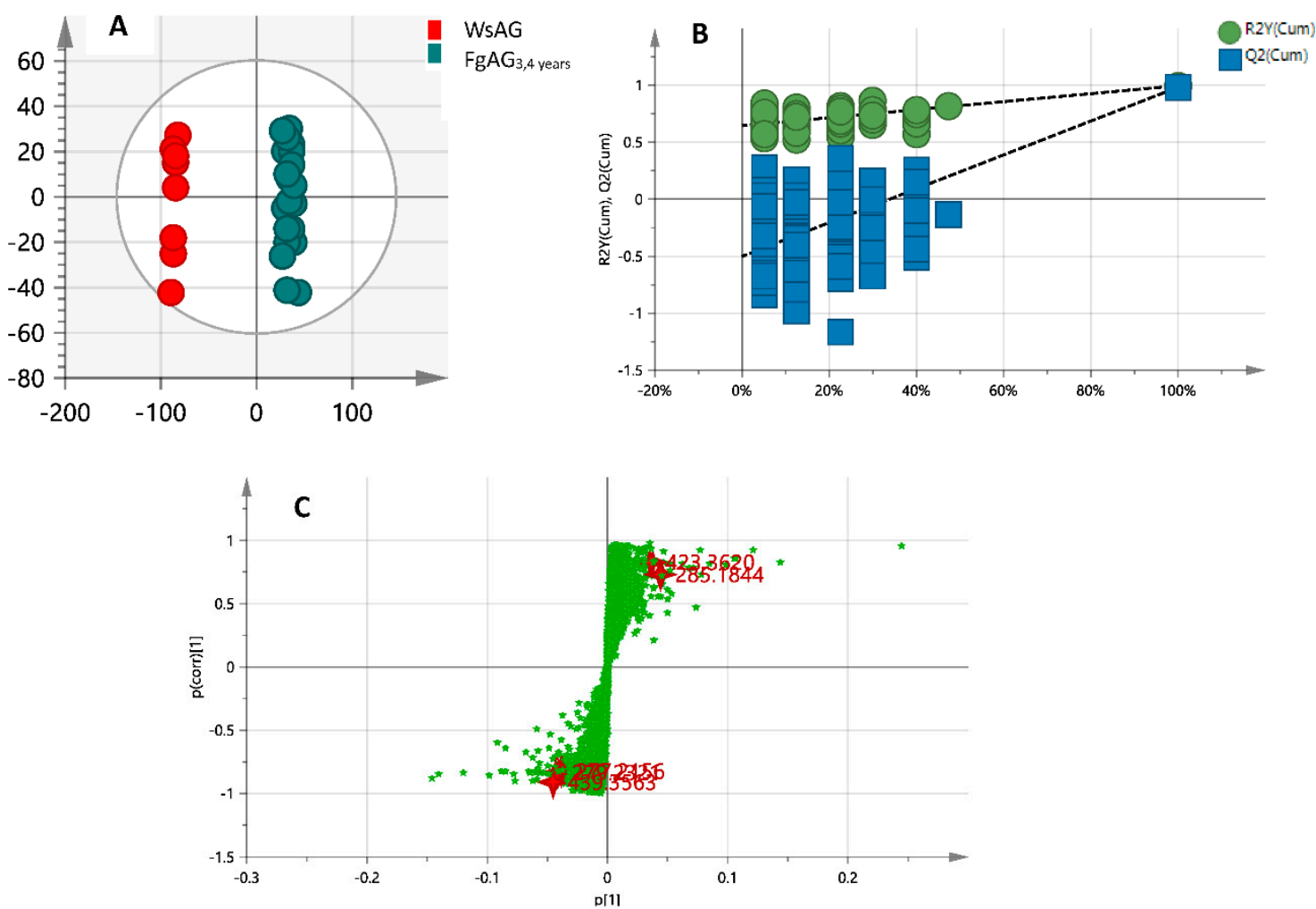

Figure 5. The OPLS-DA (A); permutation tests (B) and S-plot (C) in positive mode.

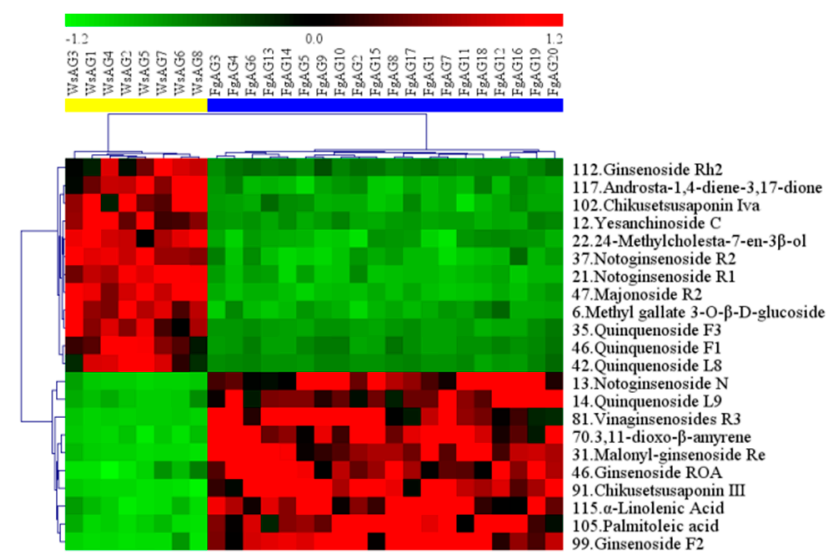

Figure 6. The heatmap visualizing the intensities of potential biomarkers.

\section{Discussion}

In the screening analysis, 121 compounds were characterized in FgAG and WsAG, respectively. The results showed that both of these kinds of American ginseng were rich in natural components. These 121 compounds were all shared constituents in FgAG and WsAG, which means that they were similar in terms of the kinds of compound they contained. It has been reported that there are high ginsenoside contents in American ginseng. In this study, ginsenosides were also the main chemical components. Besides the most common dammarane-type and oleanane-type saponins, the ocotillol-type saponins are also occupying a notable proportion. The ocotillol-type is the characteristic type of saponin enabling American ginseng to be differentiated from Asian ginseng. So far, the studies on the mechanism of biosynthesis were focused on dammarane-type and oleanane-type ginsenosides. For example, dammaranediol was obtained by DS (dammarenediol synthase), and then modified by CYP450 to obtain dammarane-type saponins. Another example, oleanane-type 
ginsenosides were obtained by modifing $\beta$-amyrin with CYP450 and UGT (UDP-glycosyltransferase). Actually, there were little literature about the mechanism of ocotillol-type ginsenoside biosynthesis. The phytochemicals in WsAG and FgAG might provide a material basis for mechanistic studies. In short, this comprehensive and unique phytochemical profile study revealed the structural diversity of secondary metabolites and the similar patterns in FgAG and WsAG.

Furthermore, in non-targeted metabolomic analysis, when taking the contents of the constituents into account, it was found that there indeed existed quite a few differences between FgAG and WsAG, and 22 robust known biomarkers enabling the differentiation were discovered. This study illustrated the differences between FgAG and WsAG, and provided a basis for explaining the effect of different growth environments on secondary metabolites. For WsAG, there are 12 potential biomarkers, including two ocotillol-type saponins $(12,47)$, two steroids $(22,117)$, six damarane-type saponins $(21,35,37,42,44,112)$, one oleanane-type saponin (102) and one other compound (6). The contents of these 12 components in WsAG were much greater than in FgAG. On the other hand, for FgAG, there are 10 potential biomarkers including two organic acids $(\mathbf{1 0 5}, \mathbf{1 1 5})$, six damarane-type saponins $(13,14,31,46,81,91,99)$, one oleanane-type saponin (46), and one ursane (70), which contents in FgAG were much greater than in WsAG. It has been reported that wild American ginseng has better biological activity than the FgAG. As is known, biological activity is caused by the high contents of phytochemicals. Correlation studies between potential markers and biological activities could be performed in the future.

Even so, there are still several unresolved issues. For example, as shown in BPI chromatograms, though 121 compounds were identified, there are still some unidentified components. Further research should be carried on based on the formula of these unknown compounds.

\section{Materials and Methods}

\subsection{Materials and Reagents}

Twenty eight batches of commercially available FgAGs and WsAGs root products were collected or purchased from different cultivation areas in China and American, including 20 batches of FgAGs and eight batches of WsAGs. A detailed sample list is provided in Table 2.

For FgAGs, six roots of each sample were selected for analysis, while for WsAGs, 2-3 roots of each sample were analyzed. All the herbs were authenticated by the authors and the corresponding voucher specimens have been deposited in the Research Center of Natural Drug, School of Pharmaceutical Sciences, Jilin University, China.

A total of 25 saponins were isolated in our laboratory and identified by spectroscopic data. Among of these saponins, ginsenoside Ro [69], 15 ginsenosides [70,71] $\left(\mathrm{Rb}_{1}, \mathrm{Rb}_{2}, \mathrm{Rb}_{3}, \mathrm{Rc}, \mathrm{Rd}\right.$, $\mathrm{Re}, 20(S)-\mathrm{Rg}_{3}, 20(R)-\mathrm{Rg}_{3}, 20(S)-\mathrm{Rh}_{2}, \mathrm{Rg}_{1}, 20(R)-\mathrm{Rg}_{2}, 20(S)-\mathrm{Rh}_{1}, 20(R)-\mathrm{Rh}_{1}$, pseudo-ginsenoside $\mathrm{F}_{11}$, pseudo-ginsenoside $\mathrm{RT}_{5}$ ) and another six saponins [71] (quinquenoside $\mathrm{L}_{8}, \mathrm{~L}_{9}, \mathrm{~L}_{11}, \mathrm{~F}_{3}$, 24(S)-pseudoginsenoside $F_{11}$, gypenoside XVII) were isolated and identified by our group.

Oleanolic acid-28-O- $\beta$-D-glucopyranoside, ginsenoside $\mathrm{Rs}_{1},-\mathrm{Rs}_{2}$ and methyl gallate-3-O- $\beta$-Dglucoside were also isolated in our laboratory and identified by NMR spectroscopy. Adenosine, $\alpha$-maltose, L-tryptophan, notoginsenoside $\mathrm{R}_{1}$, kaempferol, L-phenylalanine, sucrose, palmitoleic acid, quinic acid and $\alpha$-linolenic acid were purchased from Beijing Zhongke Quality Inspection Biotechnology Co., Ltd. (Beijing, China).

Acetonitrile and methanol suitable for UPLC-MS were purchased from Fisher Chemical Company (Geel, Belgium). Formic acid was purchased from Sigma-Aldrich Company (St. Louis, MO, USA). Deionized water was purified using a Millipore water purification system (Millipore, Billerica, MA, USA). All other chemicals were of analytical grade. 
Table 2. Details of FgAG and WsAG samples.

\begin{tabular}{|c|c|c|c|c|}
\hline Species and the Morphological Features & Source & Growth Year & Collection Time & Batch No. \\
\hline & Ji'an City, Jilin Province, China & 3,4 & 2017.09-2017.10 & FgAG1, 11 \\
\hline & Fusong County, Jilin Province, China & 3,4 & 2017.09-2017.10 & FgAG2, 12 \\
\hline & Tonghua City, Jilin Province, China & 3,4 & 2017.09-2017.10 & FgAG3,13 \\
\hline FgAGs & Jingyu Country, Jilin Province, China & 3,4 & 2017.09-2017.10 & FgAG4, 14 \\
\hline Main roots $9 \sim 15 \mathrm{~cm}$ (length) $\times 1.5 \sim 3.0 \mathrm{~cm}$ (diameter); $2 \sim 3$ branch roots with & Antu Country, Jilin Province, China & 3,4 & 2017.09-2017.10 & FgAG5, 15 \\
\hline \multirow{5}{*}{$\begin{array}{c}\text { diameters of } 2 \sim 3.5 \mathrm{~cm} \text {; fibrous roots with diameters of } 0.1 \sim 0.2 \mathrm{~cm} ; 3 \sim 4 \text { stem scars in } \\
\text { rhizomes; no adventitious roots. }\end{array}$} & Hunchun City, Jilin Province, China & 3,4 & 2017.09-2017.10 & FgAG6, 16 \\
\hline & Helong City, Jilin Province, China & 3,4 & 2017.09-2017.10 & FgAG7, 17 \\
\hline & Huadian City, Jilin Province, China & 3,4 & 2017.09-2017.10 & FgAG8, 18 \\
\hline & Huairou District, Beijing Province, China & 3,4 & 2017.10-2017.11 & FgAG9, 19 \\
\hline & Wendeng Area, Shandong Province, China & 3,4 & 2017.10-2017.11 & FgAG10, 20 \\
\hline \multirow{3}{*}{$\begin{array}{l}\text { WsAGs } \\
\text { Main roots } 5.0 \sim 6.0 \mathrm{~cm} \text { (length) } \times 1.5 \sim 2.0 \mathrm{~cm} \text { (diameter); } 2 \sim 3 \text { branch roots with } \\
\text { diameters of } 0.5 \sim 0.9 \mathrm{~cm} \text {; fibrous roots with diameters of } 0.1 \sim 0.2 \mathrm{~cm} ; 15 \sim 25 \text { stem } \\
\text { scars in rhizomes; adventitious roots with diameters of } 0.5 \sim 0.8 \mathrm{~cm}\end{array}$} & Lawton Coumtry, Michigan State, American & $>15$ & 2017.09-2017.11 & WsAG1, 3, 6 \\
\hline & Schoharie County, Catskill region, American & $>15$ & 2017.09-2017.10 & WsAG $2,4,8$ \\
\hline & Monongalia County, West Virginia, American & $>15$ & 2017.10-2017.11 & WsAG5, 7 \\
\hline
\end{tabular}




\subsection{Sample Preparation and Extraction}

All samples were respectively air-dried, grinded and sieved (Chinese National Standard Sieve No. 3, R40/3 series) to get a homogeneous powder. Then each fine powder was accurately weighed $(0.2 \mathrm{~g})$ and soaked with $10 \mathrm{~mL}$ of methanol overnight. On the second day, each powder was extracted in an ultrasonic bath (power of $250 \mathrm{~W}$, frequency of $40 \mathrm{kHz}$ ) for half an hour. After cooling to room temperature, the lost weight was replenished with methanol. After filtering through a syringe filter $(0.22 \mu \mathrm{m})$, the extracts were injected directly into the UPLC system. In addition, to ensure the stability and suitability consistency of MS analysis, a quality control (QC) sample was prepared by pooling the same volume $(50 \mu \mathrm{L})$ from every sample and five QC injections were performed randomly through the whole worklist. The volumes injected for samples and QC were all $5 \mu \mathrm{L}$ for each run.

\subsection{UPLC-QTOF-MS}

UPLC-QTOF-MS ${ }^{\mathrm{E}}$ analysis was performed on a Waters Xevo G2-XS QTOF mass spectrometer (Waters Co., Milford, MA, USA) equipped with a UPLC system through an electrospray ionization (ESI) interface. Chromatographic separation was performed on an ACQUITY UPLC BEH C 18 (100 mm $\times 2.1 \mathrm{~mm}, 1.7 \mu \mathrm{m})$ from Waters Corporation (Milford, MA, USA). The mobile phases were composed of eluent $\mathrm{A}(0.1 \%$ formic acid in water, $v / v)$ and eluent $\mathrm{B}(0.1 \%$ formic acid in acetonitrile, $v / v)$ with flow rate of $0.4 \mathrm{~mL} / \mathrm{min}$. The elution conditions applied were: $0 \rightarrow 2 \mathrm{~min}, 10 \% \mathrm{~B} ; 2 \rightarrow 26 \mathrm{~min}, 10 \sim 100 \%$ B; $26 \rightarrow 29 \mathrm{~min}, 100 \% \mathrm{~B} ; 29 \rightarrow 29.1 \mathrm{~min}, 100 \sim 10 \% \mathrm{~B} ; 29.1 \rightarrow 32 \mathrm{~min}, 10 \% \mathrm{~B}$. The temperature of the autosampler and the UPLC column manager were set at $15{ }^{\circ} \mathrm{C}$ and $30^{\circ} \mathrm{C}$ respectively. Mixtures of $90 / 10$ and 10/90 water/acetonitrile were used as the weak wash solvent and the strong wash solvent respectively. The mass spectrum was acquired from 100 to $1500 \mathrm{Da}$ in $\mathrm{MS}^{\mathrm{E}}$ mode. The positive mode conditions were: capillary voltage, $2.6 \mathrm{kV}$; cone voltage, $40 \mathrm{~V}$; source temperature, $150{ }^{\circ} \mathrm{C}$; desolvation temperature, $400{ }^{\circ} \mathrm{C}$; cone gas flow, $50 \mathrm{~L} / \mathrm{h}$; desolvation gas flow, $800 \mathrm{~L} / \mathrm{h}$. Negative mode conditions were identical to the positive mode conditions except for capillary voltage $(2.2 \mathrm{kV})$. In MS ${ }^{\mathrm{E}}$ mode, data acquisition was performed via the mass spectrometer by rapidly switching from a low-collision energy (CE) scan to a high-CE scan during a single LC run. The low energy function was set to $6 \mathrm{~V}$, while ramp collision energy of high energy function was set to $20 \sim 40 \mathrm{~V}$. Leucine enkephalin $(\mathrm{m} / z 556.2771$ in positive mode and 554.2615 in negative mode) was used as external reference of Lock Spray ${ }^{\mathrm{TM}}$ infused at a constant flow of $10 \mu \mathrm{L} / \mathrm{min}$. During acquisition, data were collected in continumn mode for the screening analysis, and in centroid mode for the metabolomics analysis.

\subsection{Chemical Information Database for the Components of FgAG and WsAG}

Besides the in-house Traditional Medicine Library in the UNIFI software, a systematic investigation of chemical components was conducted [34]. A self-built database of compounds that were isolated from FgAG and WsAG was established by searching online databases or internet search engines such as PubMed, Full-Text Database (CNKI), ChemSpider, Web of Science and Medline. Chemical information including the component name, molecular formula and structure of the components from the herbs were obtained from the database [56].

\subsection{The Screening Analysis by the UNIFI Platform}

To quickly identify the chemical compounds, the MS raw data, compressed with Waters Compression and Archival Tool v1.10, was automatically screened and identified by using the streamlined workflow of UNIFI 1.7.0 software (Waters, Manchester, UK) [30]. The parameters were as follows: the minimum peak area of 200 was set for 2D peak detection; the peak intensity of low energy over 1000 counts and the peak intensity of high energy over 200 counts were selected for $3 \mathrm{D}$ peak detection. Mass error up to $\pm 5 \mathrm{ppm}$ for identified compounds, retention time in the range of \pm 0.1 min was allowed to match the reference substance. The matching compounds would be generated predicted fragments from structure. The negative adducts containing $+\mathrm{COOH}$ and $-\mathrm{H}$ 
and positive adducts containing $+\mathrm{H}$ and $+\mathrm{Na}$ were selected in the analysis. Leucine-enkephalin was selected as the reference compound, and $[\mathrm{M}-\mathrm{H}]^{-} 554.2620$ was used for negative ion and $[\mathrm{M}+\mathrm{H}]^{+}$ 556.2766 was used for positive ion [72].

\subsection{The Metabolomics Analysis}

The raw data were processed by MarkerLynx XS V4.1 software (Waters, Milford, CT, USA) for alignment, deconvolution, data reduction, etc. [73]. A MarkerLynx processing method was firstly created, and the main parameters were as follows: retention time range 0-26 min, mass range 100-1500 Da, mass tolerance 0.10 , minimum intensity $5 \%$, mass window 0.10 , retention time window 0.20 , marker intensity threshold 2000 counts and noise elimination level 6 . After processing the data, the results could be showed in the Extended Statistics (XS) Viewer. $m / z$-RT pairs with corresponding intensities for all the detected peaks from each data file were listed. The same value of RT and $m / z$ in different batched of samples were regarded as the same component. Then, multivariate statistical analysis was performed. Firstly, principal component analysis (PCA) was used to show the pattern recognition and maximum variation aiming to obtain the overview and classification. Secondly, orthogonal projections to latent structures discriminant analysis (OPLS-DA) in both positive and negative modes was performed in order to get the maximum separation between MsAG and FgAG group and to explore the potential chemical markers that contributed to the differences. Then, S-plots was created to provide visualization of the OPLS-DA predictive component loading to facilitate model interpretation. Meawhile, variable importance for the projection (VIP) was helpful to screen the different components, and the metabolites with VIP value (>3.0) were considered as potential markers [29]. In addition, the permutation test was performed to provide reference distributions of the $\mathrm{R}^{2} / \mathrm{Q}^{2}$-values that could indicate the statistical significance [30-33]. Simca 15.0 software (Umetrics, Malmö, Sweden) was used to show the analysis results [56,74].

\section{Conclusions}

In a comprehensive and unique phytochemical profile study, a total of 121 chemical compounds with different structural types were identified from WsAG and FgAG. The structural patterns included protopanaxdiol-type saponins, protopanaxtriol-type saponins, ocotillol-type saponins, oleanane-type saponins and other glyosides, organic acid and organic acid esters, steroids, etc. The results showed that WsAG and FgAG were rich in natural components. Furthermore, these 121 compounds were all shared constituents in them, meaning that they were similar in the kinds of compounds they contain. In metabolomic analysis, it was found that there indeed existed several differences in the contents of the constituents between FgAG and WsAG, and 22 robust known biomarkers enabling the differentiation were discovered. In a word, the results will fill the data gap in the study on the chemical constituents of WsAG and provide a reference for quantitative determinations in its quality control.

Author Contributions: The individual contributions of authors are specified as following: Data curation, Investigation, Writing-original draft, H.L.; Methodology, Software, H.Z.; Formal analysis, Writing-original draft, J.T.; Formal analysis, Writing editing, H.W.; Conceptualization, Methodology, Q.D.; Investigation, F.W.; Data curation, Y.L.; Funding acquisition, P.L.; Supervision, J.L.

Funding: This research is supported by the Bethune Plan Research Project of Jilin University [Grant No. 2018B22] and the Graduate Innovation Fund of Jilin University [Grant No. 101832018C08, and the Ph.D. Interdisciplinary Research Project Funding of Jilin University [Grant No. 10183201847].

Conflicts of Interest: The authors declare that they have no conflicts of interest concerning this article.

\section{References}

1. Ko, S.K.; Cho, O.S.; Bae, H.M.; Sohn, U.D.; Im, B.O.; Cho, S.H.; Chung, S.H.; Lee, B.Y. Change of Ginsenoside Composition of Various American Ginseng Roots. J. Korean Soc. Appl. Biol. Chem. 2009, 52, 198-201. [CrossRef]

2. Lee, D.P. Production Procedures and Economics of the American Ginseng. J. Ginseng Res. 2006, 30, $172-180$. 
3. Chen, Y.J.; Zhao, Z.Z.; Chen, H.B.; Brand, E.; Yi, T.; Qin, M.J.; Liang, Z.T. Determination of ginsenosides in Asian and American ginsengs by liquid chromatography-quadrupole/time-of-flight MS: Assessing variations based on morphological characteristics. J. Ginseng Res. 2017, 41, 10-22. [CrossRef] [PubMed]

4. Nadeau, I.; Simard, R.R.; Olivier, A. The impact of lime and organic fertilization on the growth of wild-simulated American ginseng. Can. J. Plant Sci. 2003, 83, 603-609. [CrossRef]

5. Lim, W.; Mudge, K.W.; Vermeylen, F. Effects of population, age, and cultivation methods on ginsenoside content of wild American ginseng (Panax quinquefolium). J. Agric. Food Chem. 2005, 53, 8498-8505. [CrossRef]

6. Charest, P.; Dorais, M.; Gauthier, L.; Khanizadeh, S. The influence of soil preparation, seedling rates and organic mulch on the production of woods-cultivated ginseng. Acta Hortic. 2000, 523, 87-96. [CrossRef]

7. US Fish and Wildlife Service. American Ginseng. Available online: https://www.fws.gov/international/ permits /by-species/american-ginseng.html (accessed on 7 January 2019).

8. US Fish and Wildlife Service. American Ginseng Production in Woodlots. Available online: http:// digitalcommons.unl.edu/agroforestnotes / (accessed on 6 January 2019).

9. Schlag, E.M.; Mcintosh, M.S. RAPD-based assessment of genetic relationships among and within American ginseng (Panax quinquefolius L.) populations and their implications for a future conservation strategy. Genet. Resour. Crop Evol. 2012, 59, 1553-1568. [CrossRef]

10. Burkhart, E.P. American ginseng (Panax quinquefolius L.) floristic associations in Pennsylvania: Guidance for identifying calcium-rich forest farming sites. Agrofor. Syst. 2013, 87, 1157-1172. [CrossRef]

11. Zhu, W.; Han, B.; Sun, Y.; Wang, Z.Y.; Yang, X.H. Immunoregulatory effects of a glucogalactan from the root of Panax quinquefolium L. Carbohydr. Polym. 2012, 87, 2725-2729. [CrossRef]

12. Duda, R.B.; Zhong, Y.; Navas, V.; Li, M.Z.; Toy, B.R.; Alavarez, J.G. American ginseng and breast cancer therapeutic agents synergistically inhibit MCF-7 breast cancer cell growth. J. Surg. Oncol. 2015, 72, 230-239. [CrossRef]

13. Barton, D.L.; Soori, G.S.; Bauer, B.A.; Sloan, J.A.; Johnson, P.A.; Figueras, C.; Duane, S.; Mattar, B.; Liu, H.; Atherton, P.J.; et al. Pilot study of Panax quinquefolius (American ginseng) to improve cancer-related fatigue: A randomized, double-blind, dose-finding evaluation: NCCTG trial N03CA. Support. Care Cancer 2010, 18, 179-187. [CrossRef]

14. Assinewe, V.A.; Baum, B.R.; Gagnon, D.; Arnason, J.T. Phytochemistry of wild populations of Panax quinquefolius L. (North American ginseng). J. Agric. Food Chem. 2003, 51, 4549-4553. [CrossRef]

15. Vuksan, V.; Sievenpiper, J.L.; Wong, J.; Xu, Z.; Beljan-Zdravkovic, U.; Arnason, J.T.; Assinewe, V.; Stavro, M.P.; Jenkins, A.L.; Leiter, L.A.; et al. American ginseng (Panax quinquefolius L.) attenuates postprandial glycemia in a time-dependent but not dose-dependent manner in healthy individuals. Am. J. Clin. Nutr. 2001, 73, 753-758. [CrossRef]

16. Oshima, Y.; Sato, K.; Hikino, H. Isolation and hypoglycemic activity of quinquefolans A, B, and C, glycans of Panax quinquefolium roots. J. Nat. Prod. 1987, 50, 188-190. [CrossRef]

17. Vuksan, V.; Sievenpiper, J.L.; Koo, V.Y.; Francis, T.; Beljan-Zdravkovic, U.; Xu, Z.; Vidgen, E. American ginseng (Panax quinquefolius $\mathrm{L}$ ) reduces postprandial glycemia in nondiabetic subjects and subjects with type 2 diabetes mellitus. Arch. Intern. Med. 2000, 160, 1009-1013. [CrossRef]

18. Kitts, D.D.; Wijewickreme, A.N.; Hu, C. Antioxidant properties of a North American ginseng extract. Mol. Cell. Biochem. 2000, 203, 1-10. [CrossRef]

19. Li, Z.; Guo, Y.Y.; Wu, C.F.; Li, X.; Wang, J.H. Protective Effects of Pseudoginsenoside- $\mathrm{F}_{11}$ on Scopolamine-induced Memory Impairment in Mice and Rats. J. Pharm. Spharmacol. 2010, 51, 435-440. [CrossRef]

20. Robbins, C.S. Comparative Analysis of Management Regimes and Medicinal Plant Trade Monitoring Mechanisms for American Ginseng and Goldenseal. Conserv. Biol. 2000, 14, 1422-1434. [CrossRef]

21. Grubbs, H.J.; Case, M.A. Allozyme variation in American ginseng (Panax quinquefolius L.): Variation, breeding system, and implications for current conservation practice. Conserv. Genet. 2004, 5, 13-523. [CrossRef]

22. Crusesanders, J.M.; Hamrick, J.L. Genetic diversity in harvested and protected populations of wild American ginseng, Panax quinquefolius L. (Araliaceae). Am. J. Bot. 2004, 91, 540-548. [CrossRef]

23. Voort, M.E.V.D.; Mcgraw, J.B. Effects of harvester behavior on population growth rate affects sustainability of ginseng trade. Biol. Conserv. 2006, 130, 505-516. [CrossRef] 
24. Beyfuss, B. New York State Wild Simulated American Ginseng has been Selling for well over $\$ 1000$ per Pound. Available online: http:/ / www.ginsenggeek.org/new-york-state-wild-simulated-american-ginsengHas-been-selling-for-well-over-1000-per-pound/ (accessed on 18 January 2019).

25. Anderson, R.C.; Anderson, M.R.; Houseman, G. Wild American Ginseng. Native Plants J. 2002, 3, $93-105$.

26. Predy, G.N.; Goel, V.; Lovlin, R. Efficacy of an extract of North American ginseng containing poly-furanosyl-pyranosyl-saccharides for preventing upper respiratory tract infections: A randomized controlled trial. Cmaj 2005, 173, 1043-1048. [CrossRef]

27. Zhao, H.; Xu, J.; Ghebrezadik, H.; Hylands, P.J. Metabolomic quality control of commercial Asian ginseng, and cultivated and wild American ginseng using ${ }^{1} \mathrm{H}$ NMR and multi-step PCA. J. Pharm. Biomed. Anal. 2015, 114, 113-120. [CrossRef]

28. Wang, J.R.; Leung, C.Y.; Ho, H.M.; Chai, S.; Yau, L.F.; Zhao, Z.Z.; Jiang, Z.H. Quantitative Comparison of Ginsenosides and Polyacetylenes in Wild and Cultivated American Ginseng. Chem. Biodivers. 2010, 7, 975-983. [CrossRef]

29. Wang, C.Z.; Zhang, N.Q.; Wang, Z.Z.; Qi, Z.; Zhu, H.L.; Zheng, B.Z.; Li, P.Y.; Liu, J.P. Nontargeted Metabolomic Analysis of Four Different Parts of Platycodon grandiflorum Grown in Northeast China. Molecules 2017, 22, 1280. [CrossRef]

30. Wang, C.Z.; Zhang, N.Q.; Wang, Z.Z.; Qi, Z.; Zheng, B.Z.; Li, P.Y.; Liu, J.P. Rapid characterization of chemical constituents of Platycodon grandiflorum and its adulterant Adenophora stricta by UPLC-QTOF-MS/MS. J. Mass Spectrom. 2017, 52, 643-656. [CrossRef]

31. Zhang, F.X.; Li, M.; Qiao, L.R.; Yao, Z.H.; Li, C.; Shen, X.Y.; Wang, Y.; Yu, K.; Yao, X.S.; Dai, Y. Rapid characterization of Ziziphi Spinosae Semen by UPLC/Q-tof MS with novel informatics platform and its application in evaluation of two seeds from Ziziphus species. J. Pharm. Biomed. Anal. 2016, 122, 59-80. [CrossRef]

32. Deng, L.; Shi, A.M.; Liu, H.Z.; Meruva, N.; Liu, L.; Hu, H.; Yang, Y.; Huang, C.; Li, P.; Wang, Q. Identification of chemical ingredients of peanut stems and leaves extracts using UPLC-QTOF-MS coupled with novel informatics UNIFI platform. J. Mass Spectrom. 2016, 51, 1157-1167. [CrossRef]

33. Tang, J.F.; Li, W.X.; Tan, X.J.; Li, P.; Xiao, X.H.; Wang, J.B.; Zhu, M.J.; Li, X.L.; Meng, F. A novel and improved UHPLC-QTOF/MS method for the rapid analysis of the chemical constituents of Danhong Injection. Anal. Methods 2016, 8, 2904-2914. [CrossRef]

34. Wang, Y.R.; Wang, C.Z.; Lin, H.Q.; Liu, Y.H.; Li, Y.M.; Zhao, Y.; Li, P.Y.; Liu, J.P. Discovery of the Potential Biomarkers for Discrimination between Hedyotis diffusa and Hedyotis corymbosa by UPLC-QTOF/MS Metabolome Analysis. Molecules 2018, 23, 1525. [CrossRef]

35. Tan, J.; Wang, C.Z.; Zhu, H.L.; Zhou, B.S.; Xiong, L.X.; Wang, F.; Li, P.Y.; Liu, J.P. Comprehensive Metabolomics Analysis of Xueshuan Xinmaining Tablet in Blood Stasis Model Rats Using UPLC-Q/TOF-MS. Molecules 2018, 23, 1650. [CrossRef]

36. Yang, X.; Yang, L.; Xiong, A.; Li, D.; Wang, Z. Authentication of senecio scandens and s. vulgaris based on the comprehensive secondary metabolic patterns gained by uplc-dad/esi-ms. J. Pharm. Biomed. Anal. 2011, 56, 165-172. [CrossRef]

37. Wang, D.Q.; Feng, B.S.; Wang, X.B.; Yang, C.R.; Zhou, J. Further study on dammarane saponins of leaves of panax japonicus var.major collected in qinling m ountains china. Acta Pharm. Sin. 1989, 24, 633-637.

38. Zou, K.; Zhu, S.; Tohda, C.; Cai, S.; Komatsu, K. Dammarane-type triterpene saponins from Panax japonicus. J. Nat. Prod. 2002, 65, 346-351. [CrossRef]

39. Du, Z.; Li, J.; Zhang, X.; Pei, J.; Huang, L. An integrated LC-MS-based strategy for the quality assessment and discrimination of three panax species. Molecules 2018, 23, 2988. [CrossRef]

40. Feng, B.S.; Wang, X.B.; Wang, D.Q.; Yang, C.R.; Zhou, J. Dammarane saponins of Panax japonicus var. major collected in Qinling mountain, China. Acta Bot. Yunnanica 1987, 28, 633-636.

41. Meng, H.Y.; Wang, X.W.; Zhai, C.M.; Jiang, H.; Yang, C.J.; Song, Y.; Wang, Z.B. Isolation and Identification of Lignans from the Fruits of Acanthopanax Sessiliflorus. Inf. Tradit. Chin. Med. 2016, 30, 1-4.

42. Wang, L.L.; Han, L.F.; Yu, H.S.; Sang, M.M.; Liu, E.W.; Zhang, Y.; Fang, S.M.; Wang, T.; Gao, X.M. Analysis of the Constituents in "Zhu She Yong Xue Shuan Tong" by Ultra High Performance Liquid Chromatography with Quadrupole Time-of-Flight Mass Spectrometry Combined with Preparative High Performance Liquid Chromatography. Molecules 2015, 20, 20518-20537. [CrossRef] 
43. Wang, J.H. Studies on Chemical Constituents and Biological Activities of Stems and Leaves of American Ginseng. Ph.D. Thesis, Shenyang Pharmaceutical University, Shenyang, China, 1999.

44. Ye, D.Y. Extraction, Separation and Purification of Sterols from Abalone Gland. Master's Thesis, Fujian Agriculture And Forestry University, Fujian, China, 2015.

45. Liao, P.Y.; Wang, D.; Zhang, Y.J.; Yang, C.R. Dammarane-Type Glycosides from Steamed Notoginseng. J. Agric. Food Chem. 2008, 56, 1751-1756. [CrossRef]

46. Zang, Y.W. Studies on the chemical constituents of Schizonepeta mulifida (L.) Briq. China J. Chin. Mater. Med. $1989,14,44-45$.

47. Li, G.Y.; Zeng, Y.M.; Meng, H.; Li, X.; Wang, J.H. A new triterpenoid saponin from the leaves and stems of Panax quinquefolium L. Chin. Chem. Lett. 2009, 20, 1207-1210. [CrossRef]

48. Ha, L.T.; Pawlicki-Jullian, N.; Pillon-Lequart, M.; Boitel-Conti, M.; Duong, H.X.; Gontier, E. Hairy root cultures ofpanax vietnamensis, a promising approach for the production of ocotillol-type ginsenosides. Plant Cell Tissue Org. Cult. 2016, 126, 93-103. [CrossRef]

49. Wang, J.H.; Lia, W.; Sha, Y.; Tezuka, Y.; Kadota, S.; Li, X. Triterpenoid Saponins from Leaves and Stems of Panax Quinquefolium L. J. Asian Nat. Prod. Res. 2001, 3, 123-130. [CrossRef]

50. Zou, K.; Zhu, S.; Meselhy, M.R.; Tohda, C.; Cai, S.; Komatsu, K. Dammarane-Type Saponins from Panax japonicus and Their Neurite Outgrowth Activity in SK-N-SH Cells. J. Nat. Prod. 2002, 65, 1288-1292. [CrossRef]

51. Li, P.Y. Study on Chemical Constituents and Biological Activities of American Ginseng. Ph.D. Thesis, Shenyang Pharmaceutical University, Shenyang, China, 1999.

52. Liu, J.Y.; Xiao, S.Y.; Shang, W.F.; Xu, L.Z.; Yang, S.L. A new minor triterpene saponin from kaixin-san prescription. J. Asian Nat. Prod. Res. 2005, 7, 643-648. [CrossRef]

53. Yuan, J.B.; Chen, Y.; Liang, J.; Wang, C.Z.; Liu, X.F.; Yan, Z.H.; Tang, Y.; Li, J.K. Component analysis and target cell-based neuroactivity screening of Panax ginseng, by ultra-performance liquid chromatography coupled with quadrupole-time-of-flight mass spectrometry. J. Chromatogr. B 2016, 1038, 1-11. [CrossRef]

54. Lu, J.C.; Xu, B.B.; Zhang, X.Y.; Sun, Q.S. Study on chemical constituents of rhizome of Anemone raddeana. Acta Pharm. Sin. 2002, 37, 709-715.

55. Assimopoulou, A.N.; Papageorgiou, V.P. GC-MS analysis of penta-and tetra-cyclic triterpenes from resins of Pistacia species. Part I. Pistacia lentiscus var. Chia. Biomed. Chromatogr. 2005, 19, 285-311. [CrossRef]

56. Zhu, H.L.; Lin, H.Q.; Tan, J.; Wang, H.; Wu, F.L.; Dong, Q.H.; Liu, Y.H.; Li, P.Y.; Liu, J.P. UPLC-QTOF/ MS-Based Nontargeted Metabolomic Analysis of Mountain- and Garden-Cultivated Ginseng of Different Ages in Northeast China. Molecules 2019, 24, 33. [CrossRef]

57. Liang, G.Y.; Zhou, Y.; Cao, P.X.; Xu, B.X. Studies on chemical constituents of sabia schumanniana. Chin. Pharm. J. 2005, 39, 900-901.

58. Zhu, T.T.; Li, F.; Chen, B.; Deng, Y.; Wang, M.K.; Li, L.H. Studies on the saponins from the leaves of Studies on the saponins from the leaves of Panax ginseng. Chin. J. Appl. Environ. Biol. 2016, 22, 70-74.

59. Duc, N.M.; Nguyen, M.D.; Minh, N.N.T.; Kasai, R.; Ohtani, K.; Kasai, R. Saponins from Vietnamese Ginseng, Panax vietnamensis Haet Grushv. Collected in Central Vietnam. II. Chem. Pharm. Bull. 1994, 42, 115-122. [CrossRef]

60. Zhao, P.J.; Gan, F.Y.; Zhu, N.; Shen, Y.M. Studies on the Tissue Culture of Cynanchum otophyllum and Calli Chemical Constituents. Chin. Bull. Bot. 2003, 20, 565-571.

61. Yoshikawa, M.; Murakami, T.; Yashiro, K.; Yamahara, J.; Matsuda, H.; Saijoh, R.; Tanaka, O. Bioactive Saponins and Glycosides. XI. Structures of New Dammarane-Type Triterpene Oligoglycosides, Quinquenosides I, II, III IV, and V, from American Ginseng, the Roots of Panax quinquefolium L. Chem. Pharm. Bull. 1998, 46, 647-654. [CrossRef]

62. Li, S.L.; Lai, S.F.; Song, J.Z.; Qiao, C.F.; Liu, X.; Zhou, Y.; Cai, H.; Cai, B.C.; Xu, H.X. Decocting-induced chemical transformations and global quality of Du-Shen-Tang, the decoction of ginseng evaluated by UPLC-Q-TOF-MS/MS based chemical profiling approach. J. Pharm. Biomed. Anal. 2010, 53, 946-957. [CrossRef]

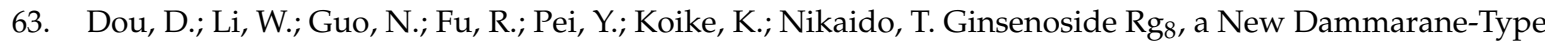
Triterpenoid Saponin from Roots of Panax quinquefolium. Chem. Pharm. Bull. 2006, 54, 751-753. [CrossRef] 
64. Ritter, A.; Goulitquer, S.; Salaün, J.P.; Tonon, T.; Correa, J.A.; Potin, P. Stress Induces Biosynthesis of Octadecanoid and Eicosanoid Oxygenated Derivatives in the Brown Algal Kelp Laminaria digitata. New Phytol. 2008, 180, 809-821. [CrossRef] [PubMed]

65. Wang, J.Y.; Li, X.G.; Zheng, Y.N.; Yang, X.W. Isoginsenoside-rh, 3 , a new triterpenoid saponin from the fruits of panax ginseng CA Mey. J. Asian Nat. Prod. Res. 2004, 6, 289-293. [CrossRef]

66. He, K.; Liu, Y.; Yang, Y.; Peng, L.; Ling, Y. A Dammarane Glycoside Derived from Ginsenoside $\mathrm{Rb}_{3}$. Chem. Pharm. Bull. 2005, 53, 177-179. [CrossRef]

67. Sun, M.; Salomon, R.G. Oxidative Fragmentation of Hydroxy Octadecadienoates Generates Biologically Active $\gamma$-Hydroxyalkenals. J. Am. Chem. Soc. 2004, 126, 5699-5708. [CrossRef] [PubMed]

68. Xu, G.H.; Choo, S.J.; Ryoo, I.J.; Kim, Y.H.; Paek, K.Y.; Yoo, I.D. Polyacetylenes from the Tissue Cultured Adventitious Roots of Panax ginseng C.A. Meyer. Nat. Prod. Sci. 2008, 14, 177-181.

69. Qiu, N.N.; Li, P.Y. Studies on the Chemical Constituents, Fingerprint and Bioactivities of Purple Red Ginseng. Master's Thesis, Jilin University, Jilin, China, 2013.

70. Liu, J.P.; Li, P.Y. Studies on Isolation, Structure Modification and Pharmacological Activities of Saponins from the Leaves and Stems of Panax quiquefolium L. Cultivated in China. Ph.D. Thesis, Shenyang Pharmaceutical University, Shenyang, China, 2005.

71. Li, P.; Liu, J.P.; Lu, D. Standard NMR Spectrum of Ginsenosides; Chemical Industry Press: Beijing, China, 2012.

72. Lee, J.W.; Ji, S.H.; Choi, B.R.; Choi, D.J.; Lee, Y.G.; Kim, H.G.; Kim, G.S.; Kim, K.; Lee, Y.H.; Baek, N.I.; et al. UPLC-QTOF/MS-Based Metabolomics Applied for the Quality Evaluation of Four Processed Panax ginseng Products. Molecules 2018, 23, 2062. [CrossRef]

73. Zhao, Y.Y.; Cheng, X.L.; Wei, F.; Xiao, X.Y.; Sun, W.J.; Zhang, Y.M.; Lin, R.C. Serum metabonomics study of adenine-induced chronic renal failure in rats by ultra performance liquid chromatography coupled with quadrupole time-of-flight mass spectrometry. Biomarkers 2012, 17, 48-55. [CrossRef] [PubMed]

74. Pang, Z.Q.; Wang, G.Q.; Ran, N.; Lin, H.Q.; Wang, Z.Y.; Guan, X.W.; Yuan, Y.Z.; Fang, K.Y.; Liu, J.P.; Wang, F. Inhibitory Effect of Methotrexate on Rheumatoid Arthritis Inflammation and Comprehensive Metabolomics Analysis Using Ultra-Performance Liquid Chromatography-Quadrupole Time of Flight-Mass Spectrometry (UPLC-Q/TOF-MS). Int. J. Mol. Sci. 2018, 19, 2894. [CrossRef] [PubMed]

Sample Availability: Samples of the compounds are not available from the authors.

(C) 2019 by the authors. Licensee MDPI, Basel, Switzerland. This article is an open access article distributed under the terms and conditions of the Creative Commons Attribution (CC BY) license (http:/ / creativecommons.org/licenses/by/4.0/). 